\title{
Organocatalytic synthesis of $\mathrm{N}$-arylaziridines
}

\author{
Sean P. Bew, ${ }^{*}$ Shirley Fairhurst, ${ }^{\S}$ David L. Hughes, Laurent Legentil, Sanket Nigudkar, Paolo Pesce \\ and Martin A. Wilson \\ "School of Chemical Sciences \& Pharmacy, University of East Anglia, Norwich, NR4 7TJ, UK; \\ ${ }^{\S}$ Department of Biological Chemistry, John Innes Centre, Norwich Research Park, NR4 7UH, UK \\ s.bew@uea.ac.uk
}

S1 - S2 Index

S3 General protocols

S4 General procedure for aldimine synthesis \& synthesis and characterization data of $\operatorname{rac}-\mathbf{8}(\mathrm{X}=\mathrm{N})$

S5 Synthesis and characterization data of $r a c-8(X=C)$

S6 Synthesis and characterization data of $r a c-8(X=C)$

S7 Synthesis and characterization data of $r a c-13$

S8 Synthesis and characterization data of $r a c-14$

S9 Synthesis and characterization data of $r a c-15$

S10 Synthesis and characterization data of $r a c-16$

S11 Synthesis and characterization data of $r a c-17$

S12 Synthesis and characterization data of $r a c-18$

S13 Synthesis and characterization data of $r a c-19$

S14 Synthesis and characterization data of $r a c-20$

S15 Synthesis and characterization data of $r a c-21$

S16 Synthesis and characterization data of rac-cis-23

S17 Synthesis and characterization data of rac-cis-24

S18 Synthesis and characterization data of $r a c-27$

S19 Synthesis and characterization data of $r a c-28$

S19 References

S20 ${ }^{1} \mathrm{H}$-NMR for $r a c-8$

S21 ${ }^{13} \mathrm{C}-\mathrm{NMR}$ for $\mathrm{rac}-\mathbf{8}$

S22 ${ }^{1} \mathrm{H}-\mathrm{NMR}$ for $r a c-13$ 
$\mathbf{S 2 3}{ }^{13} \mathrm{C}-\mathrm{NMR}$ for $r a c-13$

S24 ${ }^{1}$ H-NMR for $r a c-14$

S25 ${ }^{13}$ C-NMR for $r a c-14$

S26 ${ }^{1} \mathrm{H}-\mathrm{NMR}$ for $r a c-15$

S27 ${ }^{13}$ C-NMR for $r a c-15$

S28 ${ }^{1} \mathrm{H}-\mathrm{NMR}$ for $r a c-16$

S29 ${ }^{13} \mathrm{C}-\mathrm{NMR}$ for $r a c-16$

S30 ${ }^{1} \mathrm{H}-\mathrm{NMR}$ for $\mathrm{rac}-\mathbf{1 7}$

S31 ${ }^{13}$ C-NMR for $r a c-17$

S32 ${ }^{1} \mathrm{H}-\mathrm{NMR}$ for $r a c-18$

S33 ${ }^{1}$ H-NMR for $r a c-19$

S34 ${ }^{13}$ C-NMR for $r a c-19$

S35 ${ }^{1} \mathrm{H}-\mathrm{NMR}$ for $r a c-20$

S36 ${ }^{13} \mathrm{C}-\mathrm{NMR}$ for $r a c-20$

S37 ${ }^{1}$ H-NMR for rac-21

S38 ${ }^{13} \mathrm{C}$-NMR for $r a c-21$

S39 ${ }^{1}$ H-NMR for rac-cis-23

S40 ${ }^{13} \mathrm{C}$-NMR for $r a c-c i s-23$

S41 ${ }^{1} \mathrm{H}-\mathrm{NMR}$ for $\mathrm{rac}-27$

S42 ${ }^{13}$ C-NMR for $r a c-27$

S43 ${ }^{1}$ H-NMR for $r a c-28$

S44 ${ }^{13}$ C-NMR for rac-28

S45 HRMS for N-H pyridinium triflate and N-fluoropyridinium triflate 7 


\section{General protocols}

All reactions requiring anhydrous conditions were conducted in flame-dried glass apparatus under an atmosphere of argon. All commercially available chemicals and reagents were used as supplied.

Melting points were recorded using open capillary tubes on a Gallenkamp melting point apparatus and are uncorrected. Infrared spectra were recorded on a Perkin-Elmer Paragon 1000FT infrared spectrometer either as a thin film or neat sample. ${ }^{1} \mathrm{H}$ - and ${ }^{13} \mathrm{C}-\mathrm{NMR}$ spectra were recorded in Fourier transform mode at the field strength specified either on a Bruker AC-400 or a Varian Gemini 300 spectrometer and unless otherwise stated deuterated chloroform was used as solvent. The ${ }^{1} \mathrm{H}$-spectra were recorded in p.p.m. and referenced to the residual $\mathrm{CHCl}_{3}$ signal located at $\delta 7.26 .{ }^{13} \mathrm{C}$-NMR spectra were recorded in p.p.m. and referenced to the residual $\mathrm{CHCl}_{3}$ signal found at $\delta$ 77.00. Multiplicities in the NMR spectra are described as: $\mathrm{s}=$ singlet, $\mathrm{d}=$ doublet, $\mathrm{t}=$ triplet, $\mathrm{q}=$ quartet, $\mathrm{m}=$ multiplet, $\mathrm{br}=$ broad; coupling constants are reported in $\mathrm{Hz}$. Low resolution mass spectra were run on a either a Micromass Quattro II, Finnigan MAT95 or MAT 900 spectrometer. Ion mass/charge $(\mathrm{m} / \mathrm{z})$ ratios are reported as values in atomic mass units. Thin layer chromatography was performed on Merck aluminium plates coated with $0.2 \mathrm{~mm}$ silica gel-60 F 254 . Flash column chromatography was performed on silica gel (Kieselgel 60). 


\section{General Procedure for Aldimine Synthesis.}

To a dichloromethane solution of the starting material aldehyde was added, magnesium sulfate and amine. The solution was stirred at ambient temperature until the aldehyde was consumed. The reaction was filtered, the magnesium sulfate washed with small amount of DCM, subsequent solvent removal in vacuo, afforded the desired imines in sufficient purity ( $\left.{ }^{1} \mathrm{H}-\mathrm{NMR}\right)$ to be used 'as is' in the subsequent aziridination step.

\section{General Procedure for Aziridine Synthesis using N-fluoropyridinium Triflate.}

\section{Synthesis of $r a c-8(X=N)$}

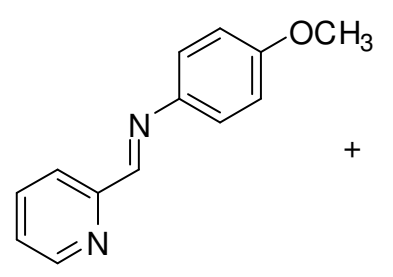

5

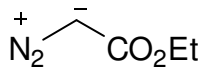

6

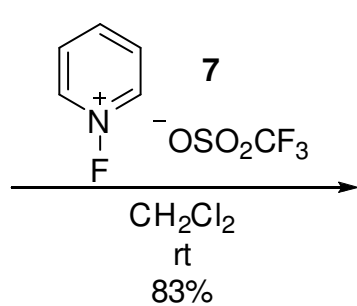

(E)-4-Methoxy- $N$-(pyridin-2-ylmethylene)phenylamine $\quad(105 \mathrm{mg}, \quad 0.50 \mathrm{mmol})$ and $\mathrm{N}$ -

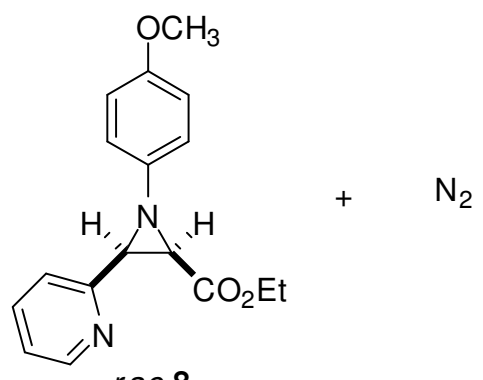

rac-8 fluoropyridinium triflate $(12 \mathrm{mg}, 0.05 \mathrm{mmol}, 10 \mathrm{~mol} \%)$ were dissolved in dichloromethane $(2 \mathrm{~mL})$. The reaction was stirred at room temperature for 5 minutes. After which time ethyl diazoacetate 6 $(0.06 \mathrm{~mL}, 0.55 \mathrm{mmol}, 1.1 \mathrm{eq})$ was added and the solution stirred at ambient temperature for a further 12 hours. Filtering the reaction through a small plug of alumina, followed by solvent removal in vacuo allowed the reaction mixture to be purified via flash column chromatography on silica gel (elution with hexane / ether / triethylamine : 80 / 20 / 1) to afford $107 \mathrm{mg}$ of rac-8 $(83 \%)$.

${ }^{1} \mathrm{H} \mathrm{NMR}\left(\mathrm{CDCl}_{3}, 400 \mathrm{MHz}\right) 8.54(\mathrm{~d}, J=4.6 \mathrm{~Hz}, 1 \mathrm{H}), 7.69\left(\mathrm{td}, J_{l}=8.8 \mathrm{~Hz}, J_{2}=2.1 \mathrm{~Hz}, 1 \mathrm{H}\right)$, $7.65(\mathrm{~d}, J=8.0 \mathrm{~Hz}, 1 \mathrm{H}), 7.21\left(\mathrm{ddd}, J_{1}=8.0 \mathrm{~Hz}, J_{2}=4.6 \mathrm{~Hz}, J_{3}=1.6 \mathrm{~Hz}, 1 \mathrm{H}\right), 6.99(\mathrm{~d}, J=8.8$ Hz, 2H), 6.81 (d, $J=8.8 \mathrm{~Hz}, 2 \mathrm{H}), 4.11-3.97(\mathrm{~m}, 2 \mathrm{H}), 3.76(\mathrm{~s}, 3 \mathrm{H}), 3.68(\mathrm{~d}, J=6.8 \mathrm{~Hz}, 1 \mathrm{H}), 3.19$ $(\mathrm{d}, J=6.8 \mathrm{~Hz}, 1 \mathrm{H}), 1.05(\mathrm{t}, J=7.2 \mathrm{~Hz}, 3 \mathrm{H}) ;{ }^{13} \mathrm{C} \mathrm{NMR}\left(\mathrm{CDCl}_{3}, 300 \mathrm{MHz}\right) \quad 167.6,156.1,155.1$, 149.0, 145.3, 136.2, 122.7, 122.6, 120.8, 114.5, 61.0, 55.4, 48.3, 45.3, 13.7; FT-IR (film, cm ${ }^{-1}$ ): 2974, 1749, 1510, 1241, 1189, 1041, 828; MS (EI) ${ }^{+}: \mathrm{m} / z 298.5(100 \%)[\mathrm{M}]^{+}$; HRMS (EI) ${ }^{+}$: exact mass calculated for $\left[\mathrm{C}_{17} \mathrm{H}_{18} \mathrm{~N}_{2} \mathrm{O}_{3}+\mathrm{H}\right]^{+}$requires $m / z$ 299.1390, found $\mathrm{m} / z$ 299.1391. 


\section{Synthesis of $r a c-8(X=C)$}

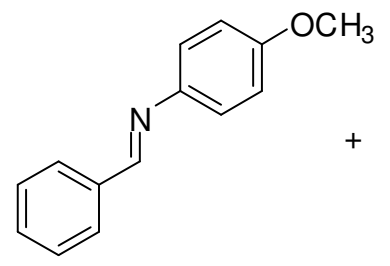

5<smiles>CCOC(=O)C[NH3+]</smiles>

6

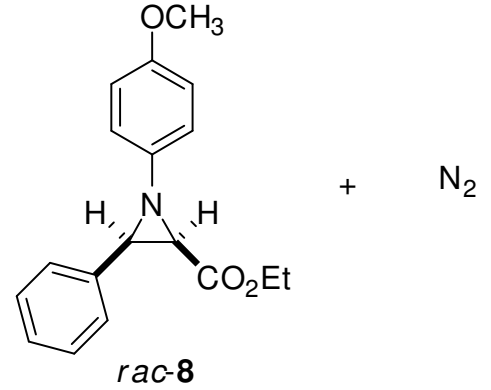

(E)- $N$-Benzylidene-4-methoxyphenylamine $(105 \mathrm{mg}, 0.5 \mathrm{mmol})$ and $\mathrm{N}$-fluoropyridinium triflate $(12 \mathrm{mg}, 10 \mathrm{~mol} \%)$ were dissolved in dichloromethane $(2 \mathrm{~mL})$. The reaction was stirred at room temperature for 5 minutes. After which ethyl diazoacetate $6(0.06 \mathrm{~mL}, 1.1 \mathrm{eq})$ was added and the solution stirred at ambient temperature for 12 hours. Filtering the reaction through a small plug of alumina, followed by solvent removal in vacuo allowed the reaction mixture to be purified via flash column chromatography on silica gel (elution with hexane / ether / triethylamine : 80 / 20 / 1) to afford $70 \mathrm{mg}(0.23 \mathrm{mmol}, 47 \%$ yield $)$ of $\mathrm{rac}-\mathbf{8}$. The title compound had physico-chemical characteristics that were essentially identical to those reported by Templeton et al. ${ }^{1}$ 


\section{Synthesis of rac-13}<smiles>COc1ccc(/N=C/c2cccnc2)cc1</smiles>

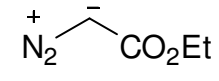

6
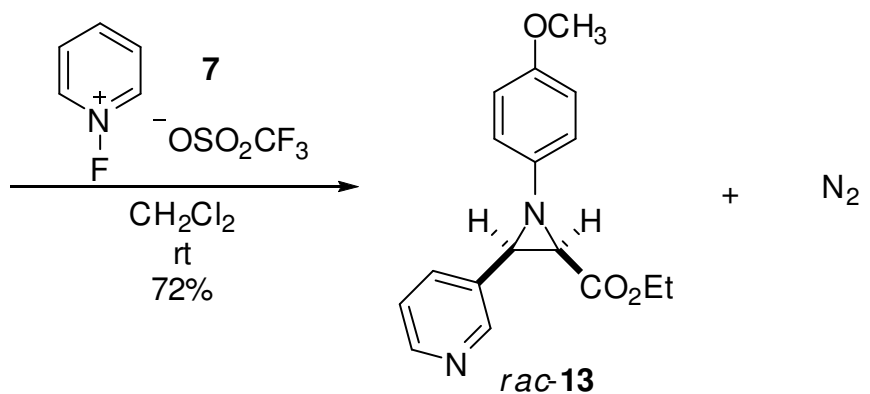

(E)-4-Methoxy- $N$-(pyridin-3-ylmethylene)phenylamine $\quad(105 \mathrm{mg}, \quad 0.50 \mathrm{mmol}) \quad$ and $\quad \mathrm{N}-$ fluoropyridinium triflate $(12 \mathrm{mg}, 0.05 \mathrm{mmol}, 10 \mathrm{~mol} \%)$ were dissolved in dichloromethane $(2 \mathrm{~mL})$. The reaction was stirred at room temperature for 5 minutes. After which ethyl diazoacetate 6 $(0.06 \mathrm{~mL}, 0.55 \mathrm{mmol}, 1.1 \mathrm{eq})$ was added and the solution stirred at ambient temperature for 12 hours. Filtering the reaction through a small plug of alumina, followed by solvent removal in vacuo allowed the reaction mixture to be purified via flash column chromatography on silica gel (elution with hexane / ether / triethylamine : 80 / 20 / 1) to afford $107 \mathrm{mg}$ of $\mathrm{rac}-\mathbf{1 3}$ (72\% yield).

${ }^{1} \mathrm{H}$ NMR $\left(\mathrm{CDCl}_{3}, 400 \mathrm{MHz}\right) 8.73(\mathrm{~d}, J=1.8 \mathrm{~Hz}, 1 \mathrm{H}), 8.55\left(\mathrm{dd}, J_{l}=4.8 \mathrm{~Hz}, J_{2}=1.8 \mathrm{~Hz}, 1 \mathrm{H}\right)$, $7.87\left(\mathrm{dt}, J_{l}=7.8 \mathrm{~Hz}, J_{2}=1.8 \mathrm{~Hz}, 1 \mathrm{H}\right), 7.29\left(\mathrm{dd}, J_{1}=7.8 \mathrm{~Hz}, J_{2}=4.8 \mathrm{~Hz}, 1 \mathrm{H}\right), 6.98(\mathrm{~d}, J=9 \mathrm{~Hz}$, $2 \mathrm{H}), 6.83(\mathrm{~d}, J=9 \mathrm{~Hz}, 2 \mathrm{H}), 4.20-3.90(\mathrm{~m}, 2 \mathrm{H}), 3.78(\mathrm{~s}, 3 \mathrm{H}), 3.52(\mathrm{~d}, J=6.6 \mathrm{~Hz}, 1 \mathrm{H}), 3.18(\mathrm{~d}, J=$ $6.6 \mathrm{~Hz}, 1 \mathrm{H}), 1.04(\mathrm{t}, J=7.2 \mathrm{~Hz}, 3 \mathrm{H}) ;{ }^{13} \mathrm{C} \mathrm{NMR}\left(\mathrm{CDCl}_{3}, 300 \mathrm{MHz}\right) 167.4,156.2,149.5,149.2$, 145.2, 135.5, 122.9, 120.7, 114.5, 61.1, 55.4, 45.5, 45.0, 13.8; FT-IR (film, cm ${ }^{-1}$ ): 3415, 1744, 1509, 1239, 1191, 1040; MS (EI) ${ }^{+}: m / z 597(100 \%)[2 \mathrm{M}+\mathrm{H}]^{+}, m / z 299(20 \%)[\mathrm{M}+\mathrm{H}]^{+} ; \mathrm{HRMS}$ $(\mathrm{EI})^{+}$: exact mass calculated for $\left[\mathrm{C}_{17} \mathrm{H}_{18} \mathrm{~N}_{2} \mathrm{O}_{3}+\mathrm{H}\right]^{+}$requires $m / z, 299.1390$, found $m / z, 299.1395$. 


\section{Synthesis of rac-14}<smiles>COc1ccc(/N=C/c2ccncc2)cc1</smiles>

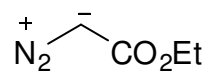

6
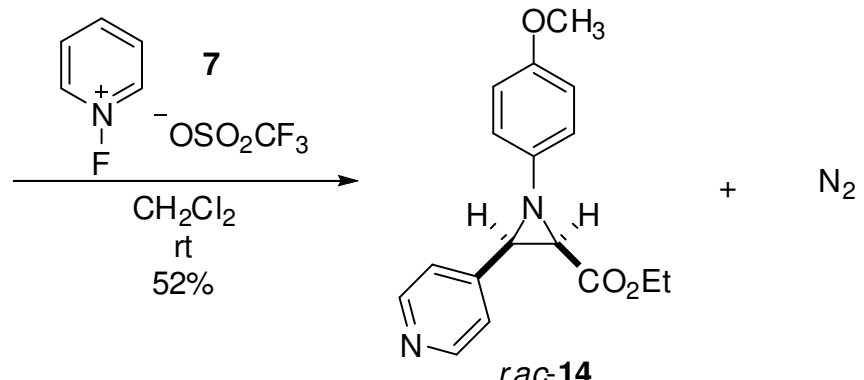

rac-14

(E)-4-Methoxy- $N$-(pyridin-4-ylmethylene)phenylamine $\quad(105 \mathrm{mg}, \quad 0.50 \mathrm{mmol}) \quad$ and $\quad \mathrm{N}-$ fluoropyridinium triflate $(12 \mathrm{mg}, 0.05 \mathrm{mmol}, 10 \mathrm{~mol} \%)$ were dissolved in dichloromethane $(2 \mathrm{~mL})$. The reaction was stirred at room temperature for 5 minutes. After which ethyl diazoacetate 6 $(0.06 \mathrm{~mL}, 0.55 \mathrm{mmol}, 1.1 \mathrm{eq})$ was added and the solution stirred at ambient temperature for 12 hours. Filtering the reaction through a small plug of alumina, followed by solvent removal in vacuo allowed the reaction mixture to be purified via flash column chromatography on silica gel (elution with hexane / ether / triethylamine : 80 / 20 / 1) to afford $78 \mathrm{mg}$ of $\mathrm{rac}-\mathbf{1 4}$ (52\% yield).

${ }^{1} \mathrm{H}$ NMR $\left(\mathrm{CDCl}_{3}, 300 \mathrm{MHz}\right) 8.58(\mathrm{~d}, J=6.0 \mathrm{~Hz}, 2 \mathrm{H}), 7.44(\mathrm{~d}, J=6.0 \mathrm{~Hz}, 2 \mathrm{H}), 6.97(\mathrm{~d}, J=9.0$ $\mathrm{Hz}, 2 \mathrm{H}), 6.82(\mathrm{~d}, J=9.0 \mathrm{~Hz}, 2 \mathrm{H}), 4.10-3.95(\mathrm{~m}, 2 \mathrm{H}), 3.78(\mathrm{~s}, 3 \mathrm{H}), 3.42$ (d, $J=6.6 \mathrm{~Hz}, 1 \mathrm{H}), 3.19$ $(\mathrm{d}, J=6.6 \mathrm{~Hz}, 1 \mathrm{H}), 1.03(\mathrm{t}, J=7.2 \mathrm{~Hz}, 3 \mathrm{H}) ;{ }^{13} \mathrm{C} \mathrm{NMR}\left(\mathrm{CDCl}_{3}, 300 \mathrm{MHz}\right)$ 167.1, 156.2, 150.0, 149.6, 144.9, 143.9, 122.8, 121.7, 120.7, 114.5, 61.2, 55.4, 45.9, 45.7, 13.8; FT-IR (film, cm ${ }^{-1}$ ): 3415, 1741, 1605, 1507, 1239, 1191, 1034; MS (EI) ${ }^{+}: m / z, 299(100 \%)[\mathrm{M}+\mathrm{H}]^{+}$; HRMS (EI) ${ }^{+}$: exact mass calculated for $\left[\mathrm{C}_{17} \mathrm{H}_{18} \mathrm{~N}_{2} \mathrm{O}_{3}+\mathrm{H}\right]^{+}$requires $m / z$ 299.1390, found $m / z, 299.1390$. 


\section{Synthesis of rac-15}
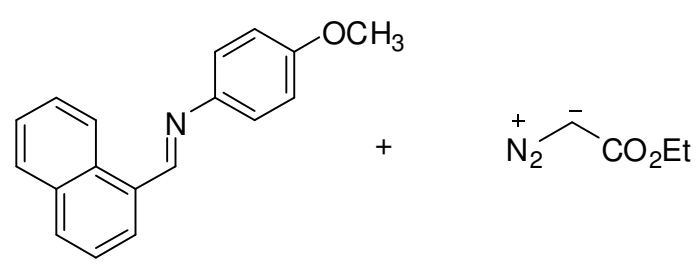

6

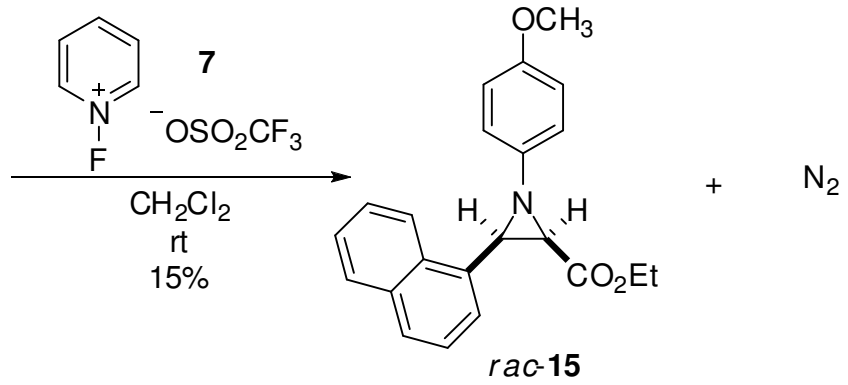

(E)-4-Methoxy- $N$-(naphthalene-1-ylmethylene)phenylamine $\quad(130 \mathrm{mg}, \quad 0.50 \mathrm{mmol})$ and $\quad N$ fluoropyridinium triflate $(12 \mathrm{mg}, 0.05 \mathrm{mmol}, 10 \mathrm{~mol} \%)$ were dissolved in dichloromethane $(2 \mathrm{~mL})$. The reaction was stirred at room temperature for 5 minutes. After which ethyl diazoacetate 6 (0.06 mL, $0.55 \mathrm{mmol}, 1.1 \mathrm{eq})$ was added and the solution stirred at ambient temperature for 12 hours. Filtering the reaction through a small plug of alumina, followed by solvent removal in vасио allowed the reaction mixture to be purified via flash column chromatography on silica gel (elution with hexane / ether / triethylamine : 80 / 20 / 1) to afford $26 \mathrm{mg}$ of $\mathrm{rac}-\mathbf{1 5}$ (15\% yield).

${ }^{1} \mathrm{H}$ NMR $\left(\mathrm{CDCl}_{3}, 400 \mathrm{MHz}\right) 8.08(\mathrm{~d}, J=7.6 \mathrm{~Hz}, 1 \mathrm{H}), 7.91-7.85(\mathrm{~m}, 2 \mathrm{H}), 7.81(\mathrm{~d}, J=8.0 \mathrm{~Hz}$, 1H), 7.50-7.30 (m, 3H), 7.08 (d, $J=8.8 \mathrm{~Hz}, 2 \mathrm{H}), 6.84$ (d, $J=8.8 \mathrm{~Hz}, 2 \mathrm{H}), 3.99$ (d, $J=6.8 \mathrm{~Hz}$, $1 \mathrm{H}), 3.90-3.70(\mathrm{~m}, 2 \mathrm{H}), 3.79(\mathrm{~s}, 3 \mathrm{H}), 3.39(\mathrm{~d}, J=6.8 \mathrm{~Hz}, 1 \mathrm{H}), 0.60(\mathrm{t}, J=6.8 \mathrm{~Hz}, 3 \mathrm{H}) ;{ }^{13} \mathrm{C} \mathrm{NMR}$ $\left(\mathrm{CDCl}_{3}, 300 \mathrm{MHz}\right) 167.9,156.0,146.0,133.3,131.4,130.6,128.7,128.2,126.4,126.2,125.8$, 125.4, 123.0, 120.9, 114.5, 60.7, 55.4, 46.0, 45.3, 13.3; FT-IR (film, $\mathrm{cm}^{-1}$ ): 2985, 1744, 1721, 1509, 1242, 1182, 1037; MS (EI) ${ }^{+}: m / z 348(100 \%)[\mathrm{M}+\mathrm{H}]^{+}, m / z 370(30)[\mathrm{M}+\mathrm{Na}]^{+}, m / z 717$ $(40 \%)[2 \mathrm{M}+\mathrm{Na}]^{+} ; \mathrm{HRMS}(\mathrm{EI})^{+}$: exact mass calculated for $\left[\mathrm{C}_{22} \mathrm{H}_{21} \mathrm{NO}_{3}+\mathrm{H}\right]^{+}$requires $\mathrm{m} / z$ 348.1594 , found $\mathrm{m} / \mathrm{z} 348.1589$. 


\section{Synthesis of rac-16}<smiles>COc1ccc(/N=C/c2ccc(Br)cc2)cc1</smiles><smiles>CCOC(=O)C[18NH2]</smiles>

6
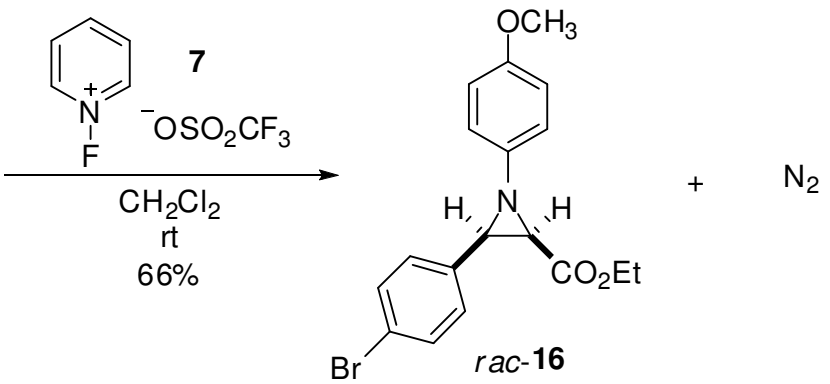

(E)-N-(4-Bromobenzylidene)-4-methoxyphenylamine $\quad(145 \mathrm{mg}, \quad 0.50 \mathrm{mmol}) \quad$ and $\mathrm{N}-$ fluoropyridinium triflate $(12 \mathrm{mg}, 0.05 \mathrm{mmol}, 10 \mathrm{~mol} \%)$ were dissolved in dichloromethane $(2 \mathrm{~mL})$. The reaction was stirred at room temperature for 5 minutes. After which ethyl diazoacetate 6 (0.06 mL, $0.55 \mathrm{mmol}, 1.1 \mathrm{eq})$ was added and the solution stirred at ambient temperature for 12 hours. Filtering the reaction through a small plug of alumina, followed by solvent removal in vacuo allowed the reaction mixture to be purified via flash column chromatography on silica gel (elution with hexane / ether / triethylamine : 80 / 20 / 1) to afford $111 \mathrm{mg}$ of rac-16 (66\% yield).

${ }^{1} \mathrm{H}$ NMR $\left(\mathrm{CDCl}_{3}, 400 \mathrm{MHz}\right) 7.47(\mathrm{~d}, J=8.7 \mathrm{~Hz}, 2 \mathrm{H}), 7.39(\mathrm{~d}, J=8.7 \mathrm{~Hz}, 2 \mathrm{H}), 6.97(\mathrm{~d}, J=8.8$ $\mathrm{Hz}, 2 \mathrm{H}), 6.81(\mathrm{~d}, J=8.8 \mathrm{~Hz}, 2 \mathrm{H}), 4.10-3.95(\mathrm{~m}, 2 \mathrm{H}), 3.78(\mathrm{~s}, 3 \mathrm{H}), 3.46(\mathrm{~d}, J=6.7 \mathrm{~Hz}, 1 \mathrm{H}), 3.13$ $(\mathrm{d}, J=6.7 \mathrm{~Hz}, 1 \mathrm{H}), 1.05(\mathrm{t}, J=7.0 \mathrm{~Hz}, 3 \mathrm{H}) ;{ }^{13} \mathrm{C} \mathrm{NMR}\left(\mathrm{CDCl}_{3}, 300 \mathrm{MHz}\right)$ 167.6, 156.0, 145.4, 133.9, 131.2, 129.4, 121.8, 120.7, 114.5, 61.0, 55.4, 46.7, 45.7, 13.8; FT-IR (film, $\mathrm{cm}^{-1}$ ): 1747, 1507, 1242, 1180, 1035; MS (EI) ${ }^{+}: m / z 376(30 \%)[\mathrm{M}+\mathrm{H}]^{+}, \mathrm{m} / z 482(100 \%)$; HRMS (EI) ${ }^{+}:$ exact mass calculated for $\left[\mathrm{C}_{18} \mathrm{H}_{18} \mathrm{BrNO}_{3}+\mathrm{H}\right]^{+}$requires $\mathrm{m} / \mathrm{z} 376.0543$, found $\mathrm{m} / \mathrm{z} 376.0545$. 


\section{Synthesis of rac-17}
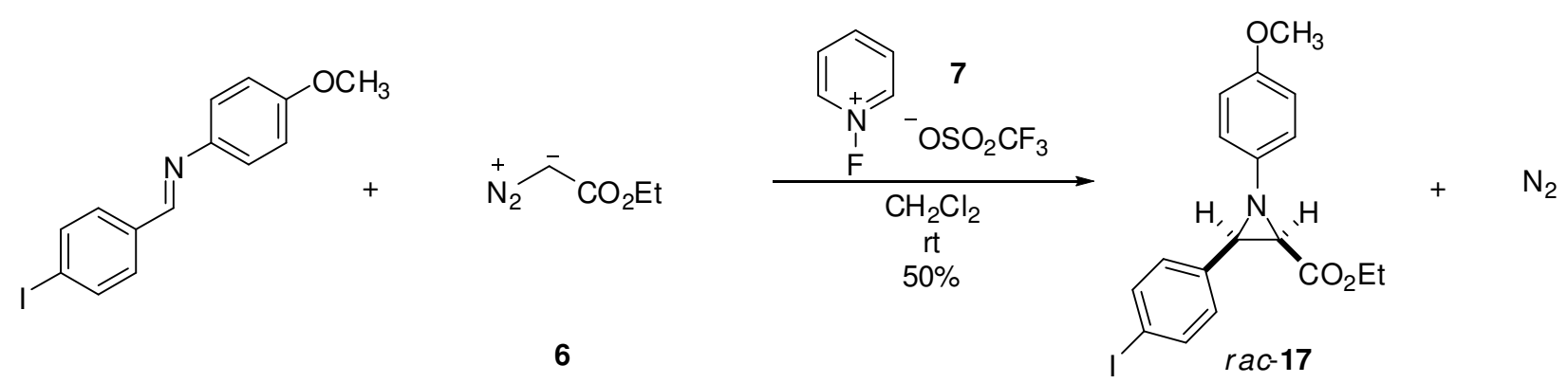

(E)-N-(4-Iodobenzylidene)-4-methoxyphenylamine (167mg, 0.50mmol) and $\mathrm{N}$-fluoropyridinium triflate $(12 \mathrm{mg}, 0.05 \mathrm{mmol}, 10 \mathrm{~mol} \%)$ were dissolved in dichloromethane $(3 \mathrm{~mL})$. The reaction was stirred at room temperature for 5 minutes. After which ethyl diazoacetate $6(0.06 \mathrm{~mL}, 0.55 \mathrm{mmol}$, $1.1 \mathrm{eq})$ was added and the solution stirred at ambient temperature for 12 hours. Filtering the reaction through a small plug of alumina, followed by solvent removal in vacuo allowed the reaction mixture to be purified via flash column chromatography on silica gel (elution with hexane / ether / triethylamine : 80 / 20 / 1) to afford $106 \mathrm{mg}$ of rac-17 (50\% yield).

${ }^{1} \mathrm{H}$ NMR $\left(\mathrm{CDCl}_{3}, 400 \mathrm{MHz}\right) 7.66(\mathrm{~d}, J=8.2 \mathrm{~Hz}, 2 \mathrm{H}), 7.24(\mathrm{~d}, J=8.2 \mathrm{~Hz}, 2 \mathrm{H}), 6.95(\mathrm{~d}, J=9.2$ $\mathrm{Hz}, 2 \mathrm{H}), 6.80(\mathrm{~d}, J=9.2 \mathrm{~Hz}, 2 \mathrm{H}), 4.10-3.90(\mathrm{~m}, 2 \mathrm{H}), 3.76(\mathrm{~s}, 3 \mathrm{H}), 3.43$ (d, $J=6.8 \mathrm{~Hz}, 1 \mathrm{H}), 3.12$ $(\mathrm{d}, J=6.8 \mathrm{~Hz}, 1 \mathrm{H}), 1.04(\mathrm{t}, J=7.0 \mathrm{~Hz}, 3 \mathrm{H}) ;{ }^{13} \mathrm{C} \mathrm{NMR}\left(\mathrm{CDCl}_{3}, 300 \mathrm{MHz}\right) 167.5,156.1,145.4$, 137.2, 134.5, 129.7, 120.7, 114.5, 93.4, 61.0, 55.4, 46.8, 45.7, 13.8; IR (film, cm ${ }^{-1}$ ): 1740, 1566, 1244, 1180; $\mathrm{MS}(\mathrm{EI})^{+}: m / z 424.0(100 \%)[\mathrm{M}+\mathrm{H}]^{+}$; HRMS (EI) ${ }^{+}$: exact mass calculated for $\left[\mathrm{C}_{18} \mathrm{H}_{18} \mathrm{NIO}_{3}+\mathrm{H}\right]^{+}$requires $m / z$ 424.0404, found $m / z 424.0407$. 


\section{Synthesis of rac-18}<smiles>COc1ccc(/N=C/c2cccc(I)c2)cc1</smiles><smiles>CCOCC[NH3+]</smiles>

6
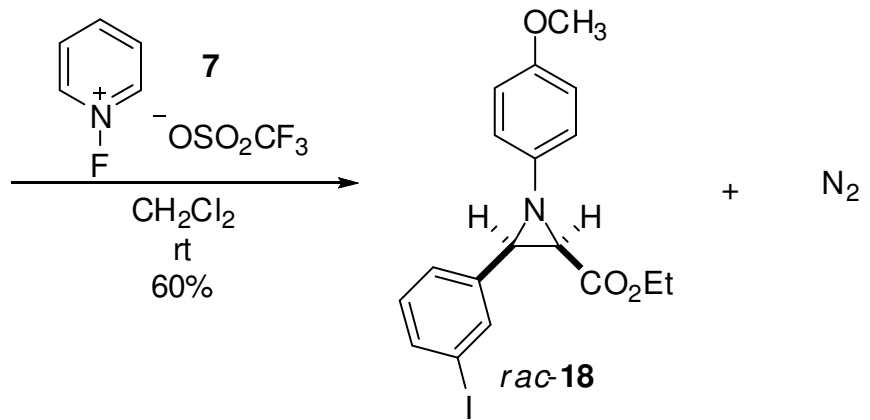

(E)-N-(3-Iodobenzylidene)-4-methoxyphenylamine (167mg, 0.50mmol) and $\mathrm{N}$-fluoropyridinium triflate $(12 \mathrm{mg}, 0.05 \mathrm{mmol}, 10 \mathrm{~mol} \%)$ were dissolved in dichloromethane $(3 \mathrm{~mL})$. The reaction was stirred at room temperature for 5 minutes. After which ethyl diazoacetate $6(0.06 \mathrm{~mL}, 0.55 \mathrm{mmol}$, $1.1 \mathrm{eq})$ was added and the solution stirred at ambient temperature for 12 hours. Filtering the reaction through a small plug of alumina, followed by solvent removal in vacuo allowed the reaction mixture to be purified via flash column chromatography on silica gel (elution with hexane / ether / triethylamine : 80 / 20 / 1) to afford $127 \mathrm{mg}$ of rac-18 (60\% yield).

${ }^{1} \mathrm{H}$ NMR $\left(\mathrm{CDCl}_{3}, 400 \mathrm{MHz}\right) 7.80(\mathrm{~s}, 1 \mathrm{H}), 7.56(\mathrm{~d}, J=8.0 \mathrm{~Hz}, 1 \mathrm{H}), 7.39(\mathrm{~d}, J=8.0 \mathrm{~Hz}, 1 \mathrm{H}), 7.03$ $(\mathrm{t}, J=8.0 \mathrm{~Hz}, 1 \mathrm{H}), 6.96(\mathrm{~d}, J=8.8 \mathrm{~Hz}, 2 \mathrm{H}), 6.75(\mathrm{~d}, J=8.8 \mathrm{~Hz}, 2 \mathrm{H}), 4.05-3.92(\mathrm{~m}, 2 \mathrm{H}), 3.71(\mathrm{~s}$, $3 \mathrm{H}), 3.38(\mathrm{~d}, J=6.8 \mathrm{~Hz}, 1 \mathrm{H}), 3.06(\mathrm{~d}, J=6.8 \mathrm{~Hz}, 1 \mathrm{H}), 0.98(\mathrm{t}, J=7.2 \mathrm{~Hz}, 3 \mathrm{H})$; FT-IR (film, cm ${ }^{-}$ $\left.{ }^{1}\right):$ 1739, 1504, 1239, 1185, 1038; MS (EI) ${ }^{+}: m / z 424(100 \%)[\mathrm{M}+\mathrm{H}]^{+}, m / z, 329(45 \%), m / z 464$ (47\%), m/z $656(70 \%)$; ) ${ }^{+}$; exact mass calculated for $\left[\mathrm{C}_{18} \mathrm{H}_{18} \mathrm{NIO}_{3}+\mathrm{H}\right]^{+}$requires $\mathrm{m} / z$ 424.0404, found $m / z$ 424.0406. 


\section{Synthesis of rac-19}

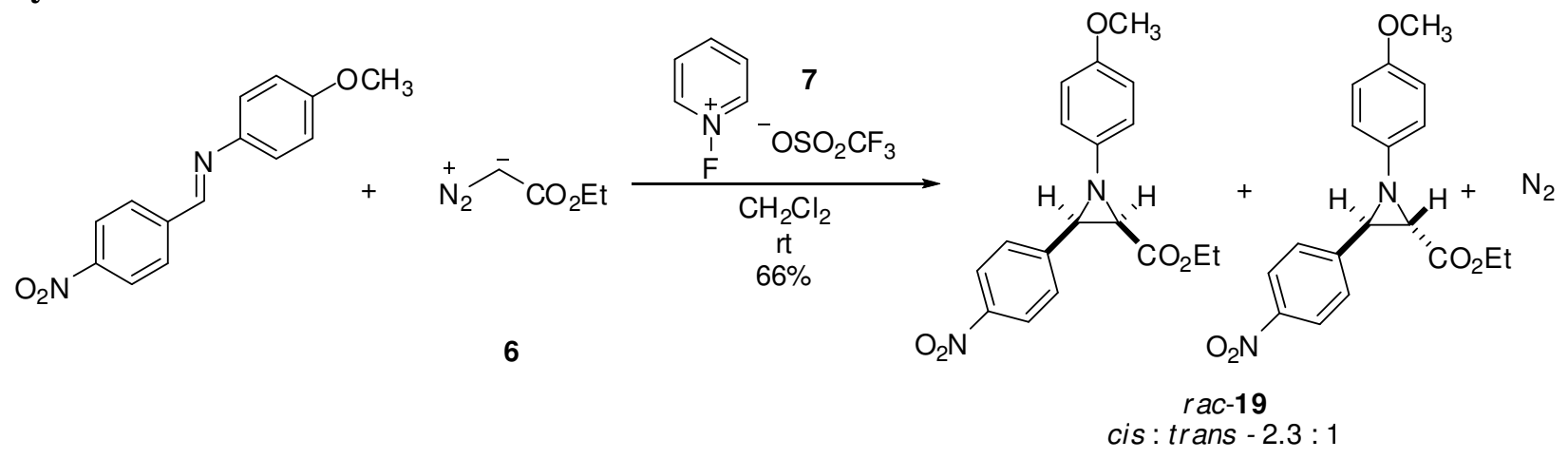

(E)-4-Methoxy- $N$-(4-nitrobenzylidene)phenylamine $(127 \mathrm{mg}, 0.50 \mathrm{mmol})$ and $N$-fluoropyridinium triflate $(12 \mathrm{mg}, 0.05 \mathrm{mmol}, 10 \mathrm{~mol} \%)$ were dissolved in dichloromethane $(3 \mathrm{~mL})$. The reaction was stirred at room temperature for 5 minutes. After which ethyl diazoacetate $6(0.06 \mathrm{~mL}, 0.55$ mmol, $1.1 \mathrm{eq}$ ) was added and the solution stirred at ambient temperature for 12 hours. Filtering the reaction through a small plug of alumina, followed by solvent removal in vacuo allowed the reaction mixture to be purified via flash column chromatography on silica gel (elution with hexane / ether / triethylamine $: 80 / 20 / 1 \rightarrow 70 / 30 / 1)$ to afford $113 \mathrm{mg}$ of rac-19 (66\% yield).

Cis-19. ${ }^{1} \mathrm{H}$ NMR $\left(\mathrm{CDCl}_{3}, 400 \mathrm{MHz}\right) 8.21(\mathrm{~d}, J=8.8 \mathrm{~Hz}, 2 \mathrm{H}), 7.70(\mathrm{~d}, J=8.8 \mathrm{~Hz}, 2 \mathrm{H}), 6.98(\mathrm{~d}, J$ $=9.0 \mathrm{~Hz}, 2 \mathrm{H}), 6.83(\mathrm{~d}, J=9.0 \mathrm{~Hz}, 2 \mathrm{H}), 4.09-3.95(\mathrm{~m}, 2 \mathrm{H}), 3.78(\mathrm{~s}, 3 \mathrm{H}), 3.57(\mathrm{~d}, J=6.7 \mathrm{~Hz}, 1 \mathrm{H})$, $3.22(\mathrm{~d}, J=6.7 \mathrm{~Hz}, 1 \mathrm{H}), 1.05(\mathrm{t}, J=7.2 \mathrm{~Hz}, 3 \mathrm{H}) ;{ }^{13} \mathrm{C} \mathrm{NMR}\left(\mathrm{CDCl}_{3}, 300 \mathrm{MHz}\right) 167.1,156.3$, 147.7, 144.9, 142.2, 128.7, 123.3, 120.7, 114.6, 61.2, 55.4, 46.4, 46.0, 13.8; FT-IR (film, cm ${ }^{-1}$ ): 2979, 1747, 1506, 1343, 1242, 1185, 1040; MS (EI) ${ }^{+}: m / z 343.1(100 \%)[\mathrm{M}+\mathrm{H}]^{+}$; HRMS (EI) ${ }^{+}$: exact mass calculated for $\left[\mathrm{C}_{18} \mathrm{H}_{18} \mathrm{~N}_{2} \mathrm{O}_{5}+\mathrm{H}\right]^{+}$requires $m / z$ 343.1288, found $m / z$, 343.1292.

Trans-19. ${ }^{1} \mathrm{H}$ NMR $\left(\mathrm{CDCl}_{3}, 400 \mathrm{MHz}\right) 8.21(\mathrm{~d}, J=8.7 \mathrm{~Hz}, 2 \mathrm{H}), 7.51(\mathrm{~d}, J=8.7 \mathrm{~Hz}, 2 \mathrm{H}), 6.83(\mathrm{~d}$, $J=8.1 \mathrm{~Hz}, 2 \mathrm{H}), 6.79(\mathrm{~d}, J=8.1 \mathrm{~Hz}, 2 \mathrm{H}), 4.13(\mathrm{q}, J=7.2 \mathrm{~Hz}, 2 \mathrm{H}), 3.86(\mathrm{~d}, J=2.4 \mathrm{~Hz}, 1 \mathrm{H}), 3.76$

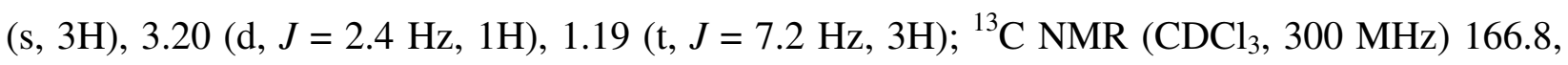
155.9, 147.8, 144.1, 140.9, 127.6, 123.7, 120.6, 114.4, 61.5, 55.3, 46.6, 45.1, 13.9; FT-IR (film, $\left.\mathrm{cm}^{-1}\right): 1732,1506,1346,1239,1182,1031 ; \mathrm{MS}(\mathrm{EI})^{+}: \mathrm{m} / \mathrm{z} 343.0(100 \%)[\mathrm{M}+\mathrm{H}]^{+}$; HRMS (EI) ${ }^{+}$: exact mass calculated for $\left[\mathrm{C}_{18} \mathrm{H}_{18} \mathrm{~N}_{2} \mathrm{O}_{5}+\mathrm{H}\right]^{+}$requires $m / z$ 343.1288, found $m / z$, 343.1290. 


\section{Synthesis of $r a c-20$}

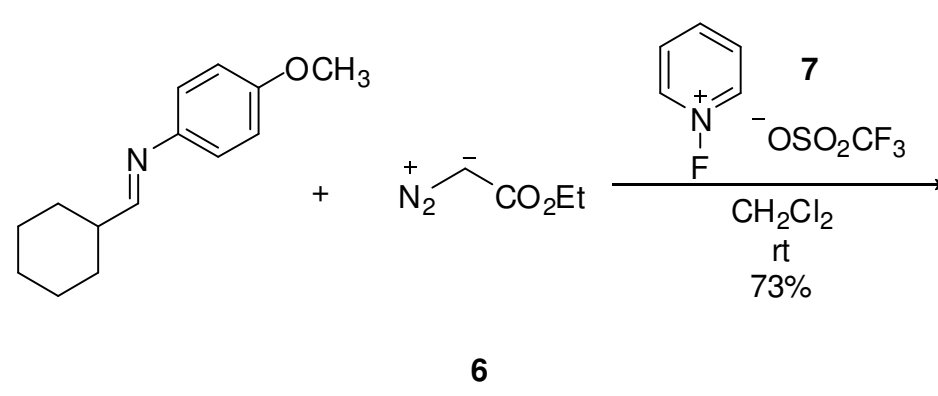

(E)-N-(Cyclohexylmethylene)-4-methoxyphenylamine

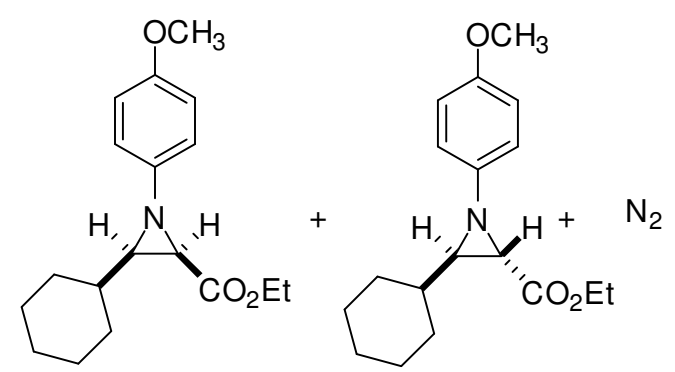

rac-20
cis : $\operatorname{trans}-1: 1$

(108mg, $0.50 \mathrm{mmol})$ and $\mathrm{N}-$

fluoropyridinium triflate $(12 \mathrm{mg}, 0.05 \mathrm{mmol}, 10 \mathrm{~mol} \%)$ were dissolved in dichloromethane $(2 \mathrm{~mL})$. The reaction was stirred at room temperature for 5 minutes. After which ethyl diazoacetate 6 (0.06 mL, $0.55 \mathrm{mmol}, 1.1 \mathrm{eq})$ was added and the solution stirred at ambient temperature for 12 hours. Filtering the reaction through a small plug of alumina, followed by solvent removal in vacuo allowed the reaction mixture to be purified via flash column chromatography on silica gel (elution with hexane / ether / triethylamine : 80 / 20 / 1) to afford $111 \mathrm{mg}$ of rac-20 (73\% yield).

Cis-20. ${ }^{1} \mathrm{H}$ NMR $\left(\mathrm{CDCl}_{3}, 400 \mathrm{MHz}\right) 6.88(\mathrm{~d}, J=8.8 \mathrm{~Hz}, 2 \mathrm{H}), 6.75(\mathrm{~d}, J=8.8 \mathrm{~Hz}, 2 \mathrm{H}), 4.35-4.25$ $(\mathrm{m}, 2 \mathrm{H}), 3.74(\mathrm{~s}, 3 \mathrm{H}), 2.78(\mathrm{~d}, J=7.0 \mathrm{~Hz}, 1 \mathrm{H}), 2.22-2.14(\mathrm{~m}, 1 \mathrm{H}), 2.10\left(\mathrm{dd}, J_{1}=9.4 \mathrm{~Hz}, J_{2}=7.0\right.$ $\mathrm{Hz}, 1 \mathrm{H}), 1.84-1.76(\mathrm{~m}, 1 \mathrm{H}), 1.74-1.62(\mathrm{~m}, 1 \mathrm{H}), 1.56-1.44(\mathrm{~m}, 2 \mathrm{H}), 1.31$ (t, $J=7.2 \mathrm{~Hz}, 3 \mathrm{H}), 1.24-$ $1.16(\mathrm{~m}, 2 \mathrm{H}), 1.14-1.04(\mathrm{~m}, 1 \mathrm{H}) ;{ }^{13} \mathrm{C} \mathrm{NMR}\left(\mathrm{CDCl}_{3}, 300 \mathrm{MHz}\right) 169.6,155.6,146.4,120.9,114.2$, 61.0, 55.4, 51.6, 42.6, 36.4, 31.8, 29.7, 26.0, 25.4, 25.3, 14.1; FT-IR (film, cm ${ }^{-1}$ ): 2924, 2846, 1747, 1506, 1443, 1244, 1178, 1098, 1041, 833; MS (EI) ${ }^{+}: m / z 303.95$ (100\%) [M] $]^{+}$; HRMS $(\mathrm{EI})^{+}$: exact mass calculated for $\left[\mathrm{C}_{18} \mathrm{H}_{25} \mathrm{NO}_{3}+\mathrm{H}\right]^{+}$requires $m / z$ 304.1907, found $m / z$ 304.1910.

Trans-20. ${ }^{1} \mathrm{H}$ NMR $\left(\mathrm{CDCl}_{3}, 400 \mathrm{MHz}\right) 6.78(\mathrm{~d}, J=10.0 \mathrm{~Hz}, 2 \mathrm{H}), 6.75(\mathrm{~d}, J=10.0 \mathrm{~Hz}, 2 \mathrm{H}), 4.07$ $3.86(\mathrm{~m}, 2 \mathrm{H}), 3.74(\mathrm{~s}, 3 \mathrm{H}), 2.82(\mathrm{~d}, J=2.6 \mathrm{~Hz}, 1 \mathrm{H}), 2.47\left(\mathrm{dd}, J_{1}=6.4 \mathrm{~Hz}, J_{2}=2.6 \mathrm{~Hz}, 1 \mathrm{H}\right), 1.92-$ $1.97(\mathrm{~m}, 1 \mathrm{H}), 1.73-1.66(\mathrm{~m}, 2 \mathrm{H}), 1.62-1.57(\mathrm{~m}, 2 \mathrm{H}), 1.21-1.11(\mathrm{~m}, 6 \mathrm{H}), 1.03(\mathrm{t}, J=7.2 \mathrm{~Hz}, 3 \mathrm{H})$;

${ }^{13} \mathrm{C} \mathrm{NMR}\left(\mathrm{CDCl}_{3}, 300 \mathrm{MHz}\right) 168.5,155.3,142.6,120.8,114.1,60.8,55.3,41.4,39.6,30.9,30.1$, 26.0, 25.6, 25.5, 13.85; FT-IR (film, $\mathrm{cm}^{-1}$ ): 2924, 2852, 1738, 1506, 1443, 1241, 1104, 1035, 830; MS (EI) $)^{+} m / z 303.95(100 \%)[\mathrm{M}]^{+}$; HRMS (EI) ${ }^{+}$: exact mass calculated for $\left[\mathrm{C}_{18} \mathrm{H}_{25} \mathrm{NO}_{3}+\right.$ $\mathrm{H}]^{+}$requires $m / z$ 304.1907, found $m / z$ 304.1905. 


\section{Synthesis of racemic-21}

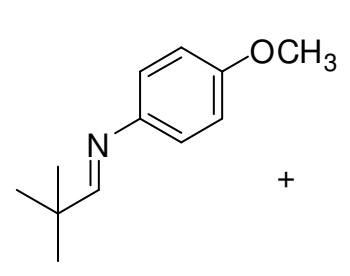

$\stackrel{+}{\mathrm{N}_{2}} \overbrace{\mathrm{CO}_{2} \mathrm{Et}}^{-}$

6

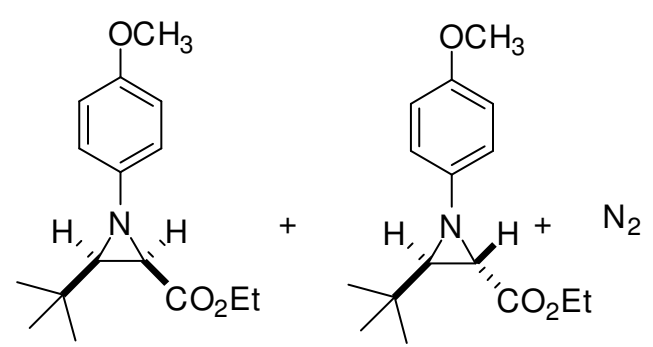

rac-21

cis : trans $-1: 1.5$

(E)- $N$-(2,2-Dimethylpropylidene)-4-methoxyphenylamine $\quad(95 \mathrm{mg}, \quad 0.50 \mathrm{mmol}) \quad$ and $\mathrm{N}$ fluoropyridinium triflate $(12 \mathrm{mg}, 0.05 \mathrm{mmol}, 10 \mathrm{~mol} \%)$ were dissolved in dichloromethane $(2 \mathrm{~mL})$. The reaction was stirred at room temperature for 5 minutes. After which ethyl diazoacetate $\mathbf{6}$ ( $0.06 \mathrm{~mL}, 0.55 \mathrm{mmol}, 1.1 \mathrm{eq}$ ) was added and the solution stirred at ambient temperature for 12 hours. Filtering the reaction through a small plug of alumina, followed by solvent removal in vacuo allowed the reaction mixture to be purified via flash column chromatography on silica gel (elution with hexane / ether / triethylamine : 80 / 20 / 1) to afford $86 \mathrm{mg}$ of $\mathrm{rac}-\mathbf{2 1}$ (62\% yield).

Cis-21. ${ }^{1} \mathrm{H}$ NMR $\left(\mathrm{CDCl}_{3}, 400 \mathrm{MHz}\right) 6.86(\mathrm{~d}, J=9.2 \mathrm{~Hz}, 2 \mathrm{H}), 6.71(\mathrm{~d}, J=9.2 \mathrm{~Hz}, 2 \mathrm{H}), 4.40-4.20$ (m, 2H), $3.75(\mathrm{~s}, 3 \mathrm{H}), 2.68(\mathrm{~d}, J=7.2 \mathrm{~Hz}, 1 \mathrm{H}), 2.13(\mathrm{~d}, J=7.2 \mathrm{~Hz}, 1 \mathrm{H}), 1.32(\mathrm{t}, J=6.8 \mathrm{~Hz}, 3 \mathrm{H})$, 1.05 (s, 9H); ${ }^{13} \mathrm{C} \mathrm{NMR}\left(\mathrm{CDCl}_{3}, 300 \mathrm{MHz}\right) 169.1,154.8,146.5,119.9,113.5,60.2,54.9,54.6$, 42.5, 30.9, 26.5, 13.2; FT-IR (film, $\mathrm{cm}^{-1}$ ): 1750, 1501, 1240, 1180, 1042; MS (EI) ${ }^{+}: \mathrm{m} / z 298$ $(100 \%)[\mathrm{M}+\mathrm{Na}]^{+}, m / z 278(95 \%)[\mathrm{M}+\mathrm{H}]^{+}, m / z 577(50 \%)[2 \mathrm{M}+\mathrm{H}]^{+}$; HRMS $(\mathrm{EI})^{+}$: exact mass calculated for $\left[\mathrm{C}_{16} \mathrm{H}_{23} \mathrm{NO}_{3}+\mathrm{H}\right]^{+}$requires $m / z$ 278.1751, found $m / z 278.1753$.

Trans-21. ${ }^{1} \mathrm{H} \mathrm{NMR}\left(\mathrm{CDCl}_{3}, 400 \mathrm{MHz}\right) 6.77(\mathrm{~d}, J=10.0 \mathrm{~Hz}, 2 \mathrm{H}), 6.75(\mathrm{~d}, J=10.0 \mathrm{~Hz}, 2 \mathrm{H}), 4.12-$ $3.97(\mathrm{~m}, 2 \mathrm{H}), 3.74(\mathrm{~s}, 3 \mathrm{H}), 2.97$ (d, $J=2.4 \mathrm{~Hz}, 1 \mathrm{H}), 2.60(\mathrm{~d}, J=2.4 \mathrm{~Hz}, 1 \mathrm{H}), 1.10$ (t, $J=7.0 \mathrm{~Hz}$, $3 \mathrm{H}), 1.01$ (s, 9H); ${ }^{13} \mathrm{C} \mathrm{NMR}\left(\mathrm{CDCl}_{3}, 300 \mathrm{MHz}\right) 168.4,155.2,143.2,120.5,114.1,60.7,55.3$, 54.2, 39.2, 30.6, 26.6, 13.8; FT-IR (film, $\mathrm{cm}^{-1}$ ): 1739, 1505, 1244, 1178; MS (EI) ${ }^{+}: \mathrm{m} / \mathrm{z} 298$ $(100 \%)[\mathrm{M}+\mathrm{Na}]^{+}, m / z 278(95 \%)[\mathrm{M}+\mathrm{H}]^{+}, m / z 332(55 \%), m / z 577(50 \%)[2 \mathrm{M}+\mathrm{H}]^{+} ; \mathrm{HRMS}$ $(\mathrm{EI})^{+}$: exact mass calculated for $\left[\mathrm{C}_{16} \mathrm{H}_{23} \mathrm{NO}_{3}+\mathrm{H}\right]^{+}$requires $m / z 278.1751$, found $m / z 278.1751$. 


\section{Synthesis of rac-cis-23}

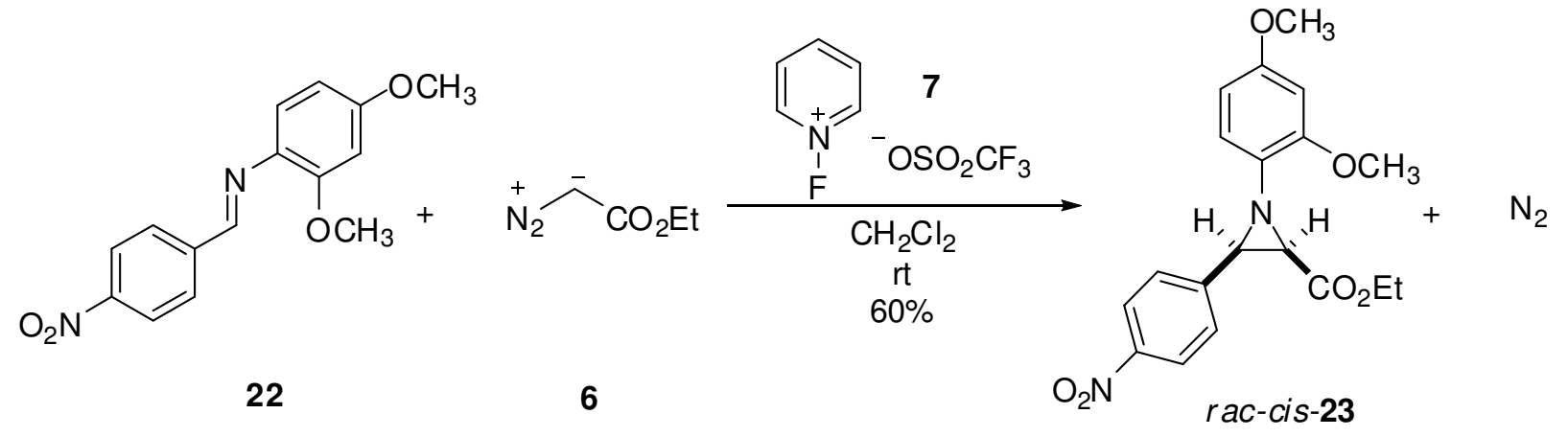

(E)-2,4-Dimethoxy- $N$-(4-nitrobenzylidene)phenylamine $\quad(1.68 \mathrm{~g}, \quad 5.86 \mathrm{mmol}) \quad$ and $\quad \mathrm{N}-$ fluoropyridinium triflate $(145 \mathrm{mg}, 0.58 \mathrm{mmol}, 10 \mathrm{~mol} \%)$ were dissolved in dichloromethane (23 $\mathrm{mL})$. The reaction was stirred at room temperature for 5 minutes. After which ethyl diazoacetate $6(0.7 \mathrm{~mL}, 6.45 \mathrm{mmol}, 1.1 \mathrm{eq})$ was added and the solution stirred at ambient temperature for 12 hours. Filtering the reaction through a small plug of alumina, followed by solvent removal in vасио allowed the reaction mixture to be purified via flash column chromatography on silica gel (eluition with hexane / ether : 4 / 1) to afford $1.3 \mathrm{~g}$ of rac-cis-23 (60\% yield).

${ }^{1} \mathrm{H}$ NMR (300 MHz, $\left.\mathrm{CDCl}_{3}\right): \delta 8.09$ (d, 2H, J = 8.44 Hz), $7.65(\mathrm{~d}, 2 \mathrm{H}, \mathrm{J}=8.41 \mathrm{~Hz}), 6.75(\mathrm{~d}, 1 \mathrm{H}$, $\mathrm{J}=8.44 \mathrm{~Hz}), 6.38(\mathrm{~s}, 1 \mathrm{H}), 6.29(\mathrm{~d}, 1 \mathrm{H}, \mathrm{J}=8.43 \mathrm{~Hz}), 3.98(\mathrm{q}, 1 \mathrm{H}, \mathrm{J}=7.08 \mathrm{~Hz}), 3.89$ (q, 1H, J = $7.10 \mathrm{~Hz}), 3.68$ (s, 3H), 3.67 (s, 3H), $3.45(\mathrm{~d}, 1 \mathrm{H}, \mathrm{J}=6.63 \mathrm{~Hz}), 3.05(\mathrm{~d}, 1 \mathrm{H}, \mathrm{J}=6.66 \mathrm{~Hz}), 0.94$ (t, $3 \mathrm{H}, \mathrm{J}=7.08 \mathrm{~Hz}) .{ }^{13} \mathrm{C} \mathrm{NMR}\left(300 \mathrm{MHz}, \mathrm{CDCl}_{3}\right): \delta 166.4,155.9,151.8,146.3,141.8,132.6$, 127.9, 126.4, 122.0, 118.6, 102.6, 98.5, 60.1, 54.6, 54.5, 46.0, 45.2, 13.0. FT-IR (film, cm ${ }^{-1}$ ): 2963, 1746, 1592, 1506, 1437, 1341, 1185, 1120, 1031; MS (EI) ${ }^{+}: m / z, 373.1(100 \%)[\mathrm{M}+\mathrm{H}]^{+}$; HRMS $(\mathrm{EI})^{+}$: exact mass calculated for $\left[\mathrm{C}_{19} \mathrm{H}_{20} \mathrm{~N}_{2} \mathrm{O}_{6}+\mathrm{H}\right]^{+}$requires $\mathrm{m} / \mathrm{z}$ 373.1394, found $\mathrm{m} / \mathrm{z}$ 373.1391 . 


\section{Synthesis of rac-cis-24}

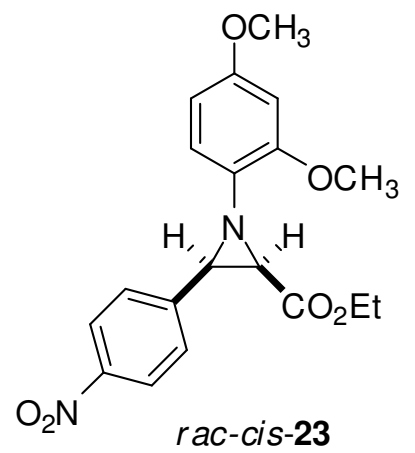

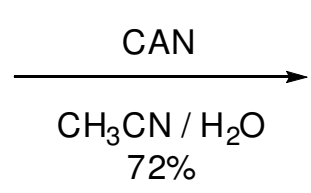

rac-cis-23

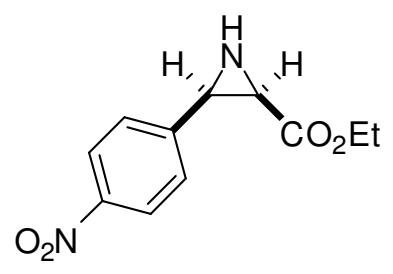

rac-cis-24

Ammonium cerium(IV) nitrate (1.2g, 2.19mmol, 4 equiv.) was dissolved in water (4 mL) and slowly added to an ice-cold solution of rac-cis-23 $(204 \mathrm{mg}, 0.55 \mathrm{mmol})$ in acetonitrile $(8 \mathrm{~mL})$. The reaction was stirred until TLC indicated consumption of the starting material. The resulting solution was concentrated under reduced pressure $\left(35^{\circ} \mathrm{C}\right.$ bath temperature). Purification via flash column chromatography using silica gel (eluition with ether / hexane : 2/1) afforded rac-cis-24 as a yellow solid in $72 \%$ yield. The physico-chemical characteristics were essentially identical to those reported by Wulff et al. ${ }^{2}$

${ }^{1} \mathrm{H}$ NMR (300 MHz, CD $\left.3 \mathrm{CN}\right): \delta 8.13(\mathrm{~d}, 2 \mathrm{H}, \mathrm{J}=8.8 \mathrm{~Hz}), 7.60(\mathrm{~d}, 2 \mathrm{H}, \mathrm{J}=8.7 \mathrm{~Hz}), 3.88(\mathrm{q}, 2 \mathrm{H}, \mathrm{J}$ $=7.0 \mathrm{~Hz}), 3.60(\mathrm{~d}, 1 \mathrm{H}, \mathrm{J}=6.4 \mathrm{~Hz}), 3.10(\mathrm{~d}, 1 \mathrm{H}, \mathrm{J}=6.4 \mathrm{~Hz}), 1.3(\mathrm{~d}, 1 \mathrm{H}, \mathrm{J}=6.3 \mathrm{~Hz}), 0.90$ (t, 3H, J $=7.0 \mathrm{~Hz}) .{ }^{13} \mathrm{C} \mathrm{NMR}\left(300 \mathrm{MHz}, \mathrm{CD}_{3} \mathrm{CN}\right): \delta 168.5,147.5,144.6,129.1,123.0,60.7,37.9,29.6$, 13.7; FT-IR (film, $\mathrm{cm}^{-1}$ ): 2983, 1732, 1602, 1519, 1344, 1201, 1108, 1026, 852, 741. 


\section{Synthesis of rac-cis-24 via imine 25}

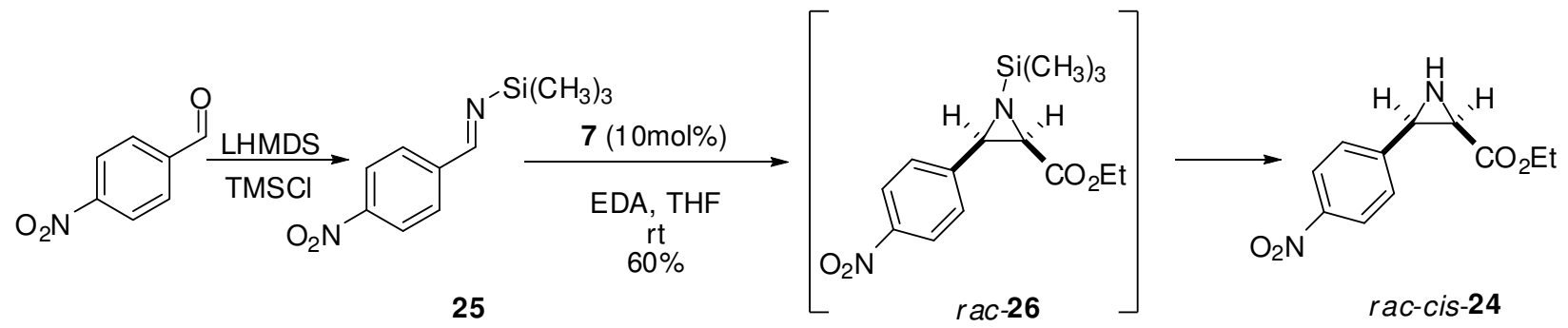

To a solution of 4-nitrobenzaldehyde $(1.0 \mathrm{~g}, 6.66 \mathrm{mmol})$ in $\mathrm{THF}(5 \mathrm{~mL})$ at $0^{\circ} \mathrm{C}$ was added lithium bis(trimethylsilyl)amide $1.06 \mathrm{M}$ in THF $\left(6.28 \mathrm{ml}, 6.28 \mathrm{mmol}, 0.95\right.$ equiv) dropwise at $0^{\circ} \mathrm{C}$. The mixture was allowed to warm to room temperature, stirred for 2 hours and then treated with chlorotrimethylsilane $(6.66 \mathrm{mmol}, 0.85 \mathrm{~mL})$. After 1 hour, anhydrous hexane was added resulting in a white precipitate. The solution was filtered under argon through dry $\mathrm{Na}_{2} \mathrm{SO}_{4}$ and washed with a small amount of anhydrous hexane. The solvents were removed in vacuo and the impure TMS-imine $(1.0 \mathrm{~g}, \sim 4.5 \mathrm{mmol})$ redissolved in dry THF $(6.5 \mathrm{~mL})$. To this solution was added EDA $(0.52 \mathrm{~mL}, 5.0 \mathrm{mmol}, 1.1$ equiv.) and a catalytic amount of $N$-fluoropyridinum triflate $(111 \mathrm{mg}, 0.45$ mmol, 0.1 equiv.). After stirring the solution at room temperature for 12 hours, the solvent was removed in vасио and the impure product purified by flash column chromatography on silica (gradient elution: pet. ether / ethyl acetate $=9 / 1 \rightarrow 4 / 1$ ). Rac-cis-24 was afforded as a yellow solid in $20 \%$ overall yield from 4-nitrobenzaldehyde. The physico-chemical characteristics were essentially identical to those reported by Wulff et al. ${ }^{2}$

${ }^{1} \mathrm{H}$ NMR (300 MHz, CD 3 CN): $\delta 8.13(\mathrm{~d}, 2 \mathrm{H}, \mathrm{J}=8.8 \mathrm{~Hz}), 7.60(\mathrm{~d}, 2 \mathrm{H}, \mathrm{J}=8.7 \mathrm{~Hz}), 3.88(\mathrm{q}, 2 \mathrm{H}, \mathrm{J}$ = 7.0 Hz), 3.60 (d, 1H, J = 6.4 Hz), 3.10 (d, 1H, J = 6.4 Hz), 1.3 (d, 1H, J = 6.3 Hz), 0.90 (t, 3H, J $=7.0 \mathrm{~Hz}) .{ }^{13} \mathrm{C} \mathrm{NMR}\left(300 \mathrm{MHz}, \mathrm{CD}_{3} \mathrm{CN}\right): \delta .168 .5,147.5,144.6,129.1,123.0,60.7,37.9,29.6$, 13.7; FT-IR (film, cm ${ }^{-1}$ ): 2983, 1732, 1602, 1519, 1344, 1201, 1108, 1026, 852, 741. 


\section{Synthesis of rac-cis-27}<smiles>CCOC1N[C@H]1c1ccc([N+](=O)[O-])cc1</smiles>

rac-cis-24

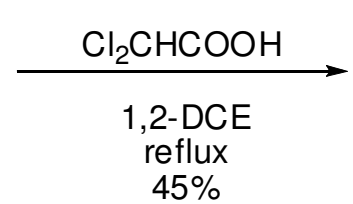

$45 \%$<smiles>CCOC(=O)C(NC(=O)CC)C(O)c1ccc([N+](=O)[O-])cc1</smiles>

rac-27

To a solution of rac-cis-24 (482 $\mathrm{mg}, 1.2 \mathrm{mmol})$ in $5 \mathrm{~mL}$ of 1,2-dichloroethane was added dichloroacetic acid $(0.1 \mathrm{~mL}, 1.2 \mathrm{mmol}, 1$ equiv.) and the mixture was heated to reflux and monitored via TLC until no more rac-cis-24 could be detected. The solvent was removed in vacuo and the residue dissolved in dichloromethane and treated with a saturated aqueous solution of sodium carbonate. The aqueous layer was extracted with dichloromethane and the combined organic extracts were dried over $\mathrm{MgSO}_{4}$. Purification by chromatography on silica gel (hexane / diethyl ether : 1 / 2) afforded rac-27 as a light yellow solid in 45\% yield. The physico-chemical characteristics were essentially identical to those reported by Wulff et al. ${ }^{3}$

${ }^{1} \mathrm{H}$ NMR (300 MHz, $\left.\mathrm{CD}_{3} \mathrm{CN}\right): \delta 8.21(\mathrm{~d}, 2 \mathrm{H}, \mathrm{J}=8.2 \mathrm{~Hz}), 7.60(\mathrm{~d}, 2 \mathrm{H}, \mathrm{J}=8.2 \mathrm{~Hz}), 7.28(\mathrm{~d}, 1 \mathrm{H}, \mathrm{J}$ $=2.7 \mathrm{~Hz}), 6.05(\mathrm{~s}, 1 \mathrm{H}), 5.50(\mathrm{~d}, 1 \mathrm{H}, \mathrm{J}=2.4 \mathrm{~Hz}), 4.79\left(\mathrm{dd}, 1 \mathrm{H}, \mathrm{J}_{1}=8.9 \mathrm{~Hz}, \mathrm{~J}=2.8 \mathrm{~Hz}\right), 4.30$ (dd, $\left.1 \mathrm{H}, \mathrm{J}_{1}=8.9 \mathrm{~Hz}, \mathrm{~J}_{2}=2.3 \mathrm{~Hz}\right), 4.21(\mathrm{q}, 2 \mathrm{H}, \mathrm{J}=7.2 \mathrm{~Hz}), 1.23(\mathrm{t}, 3 \mathrm{H}, \mathrm{J}=7.0 \mathrm{~Hz}) .{ }^{13} \mathrm{C} \mathrm{NMR}(300$ MHz, CDCN): $\delta$. 168.9, 164.2, 148.3, 147.9, 127.6, 123.4, 71.7, 66.2, 61.9, 58.5, 13.4; FT-IR (film, $\mathrm{cm}^{-1}$ ): 2926, 1742, 1683, 1608, 1517, 1348, 1206, 1110, 1074, 1015, 812. 


\section{Synthesis of $r a c-28$}<smiles>CCOC(=O)C(NC(=O)CCl)C(O)c1ccc([N+](=O)[O-])cc1</smiles>

rac-27

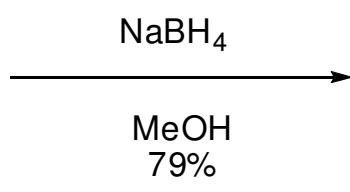

$79 \%$<smiles>O=C(CCl)NC(CO)C(O)c1ccc([N+](=O)[O-])cc1</smiles>

rac-28

Rac-27 (102mg, 0.28mmol) was dissolved in $0.5 \mathrm{~mL}$ of methanol and cooled to $0^{\circ} \mathrm{C}$. Sodium borohydride (53mg, 1.4mmol, 6 equiv.) was added to the solution all at once and the reaction mixture was stirred for 30 minutes. The product was quenched with water $(10 \mathrm{~mL})$ and extracted with ethyl acetate three times. The combined organic phase were dried over $\mathrm{MgSO}_{4}$. Purification via flash chromatography on silica gel (hexane / ethyl acetate : 3 / 7) afforded the rac-28 as a white solid in $79 \%$ yield. The physico-chemical characteristics were essentially identical to those reported by Wulff et al. ${ }^{3}$

${ }^{1} \mathrm{H}$ NMR (300 MHz, DMSO): $\delta 8.32(\mathrm{~d}, 1 \mathrm{H}, \mathrm{J}=9.0 \mathrm{~Hz}), 8.15(\mathrm{~d}, 2 \mathrm{H}, \mathrm{J}=8.7 \mathrm{~Hz}), 7.56(\mathrm{~d}, 2 \mathrm{H}, \mathrm{J}$ $=8.7 \mathrm{~Hz}), 6.46(\mathrm{~s}, 1 \mathrm{H}), 6.06\left(\right.$ broad s, 1H), $5.04\left(\right.$ broad s, 1H), 4.97 (broad s, 1H), $3.92\left(\mathrm{q}, 1 \mathrm{H}, \mathrm{J}_{1}\right.$ $=8.1 \mathrm{~Hz}), 3.57(\mathrm{t}, 1 \mathrm{H}, \mathrm{J}=8.1 \mathrm{~Hz}), 3.34(\mathrm{~m}, 1 \mathrm{H}) .{ }^{13} \mathrm{C}$ NMR $(300 \mathrm{MHz}, \mathrm{DMSO}): \delta$ 163.6, 151.5, 146.6, 127.5, 123.1, 69.1, 66.5, 60.3, 56.9; FT-IR (film, $\mathrm{cm}^{-1}$ ): 3298, 2924, 1682, 1514, 1348, 1070,812 .

\section{References}

1. L. Casarrubios, J. A. Perez, M. Brookhart, J. L. Templeton, J. Org. Chem., 1996, 61, 8358

2. Z. Lu, Y. Zhang, W. D. Wulff, J. Am. Chem. Soc., 2007, 129, 7185

3. C. Loncaric and W. D. Wulff, Org. Lett., 2001, 3, 3675 
${ }^{1} \mathrm{H}-\mathrm{NMR}$ for $r a c-8$

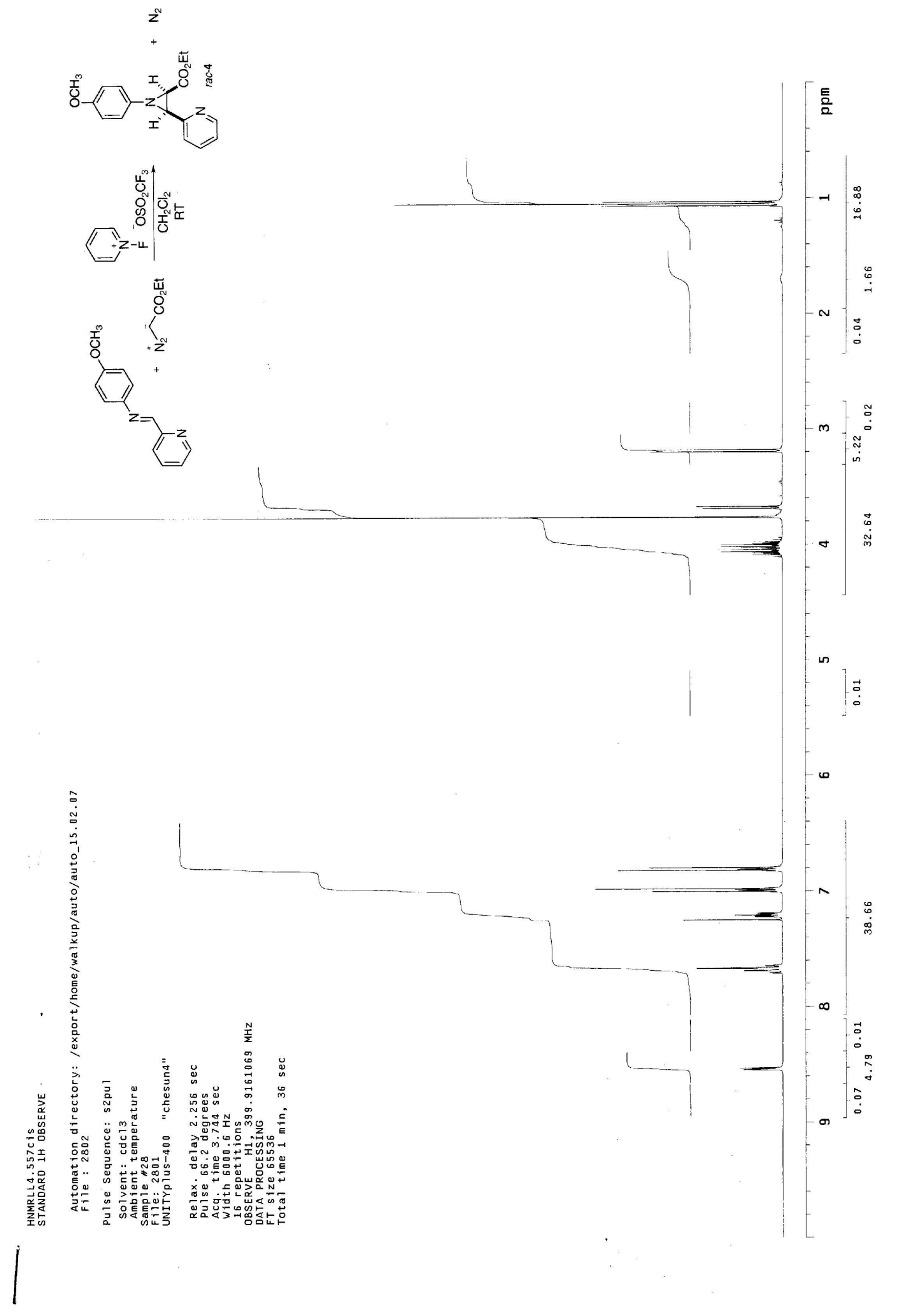




\section{${ }^{13} \mathrm{C}-\mathrm{NMR}$ for $r a c-8$}

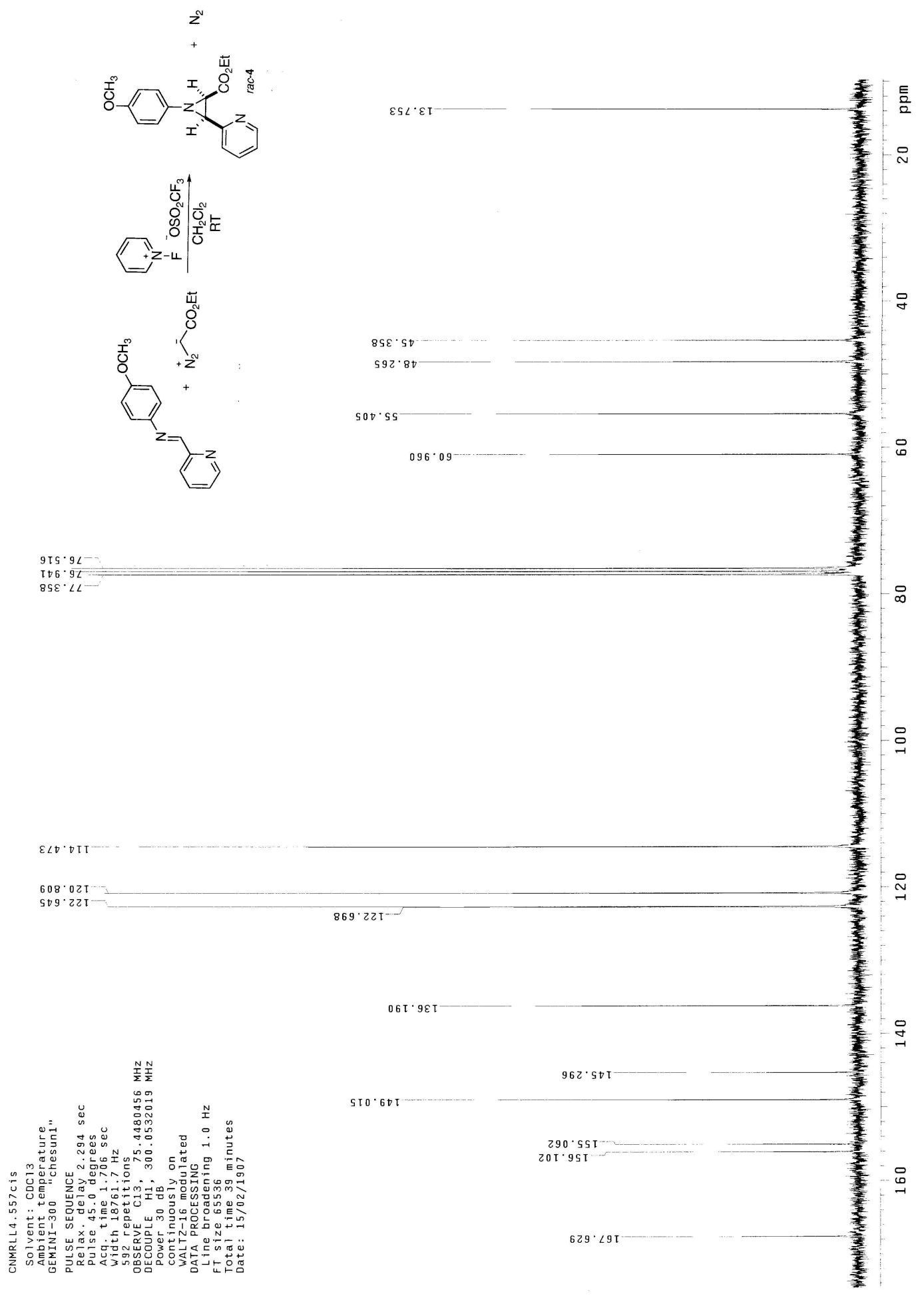




\section{${ }^{1} \mathrm{H}-\mathrm{NMR}$ for $\mathrm{rac}-\mathbf{1 3}$}

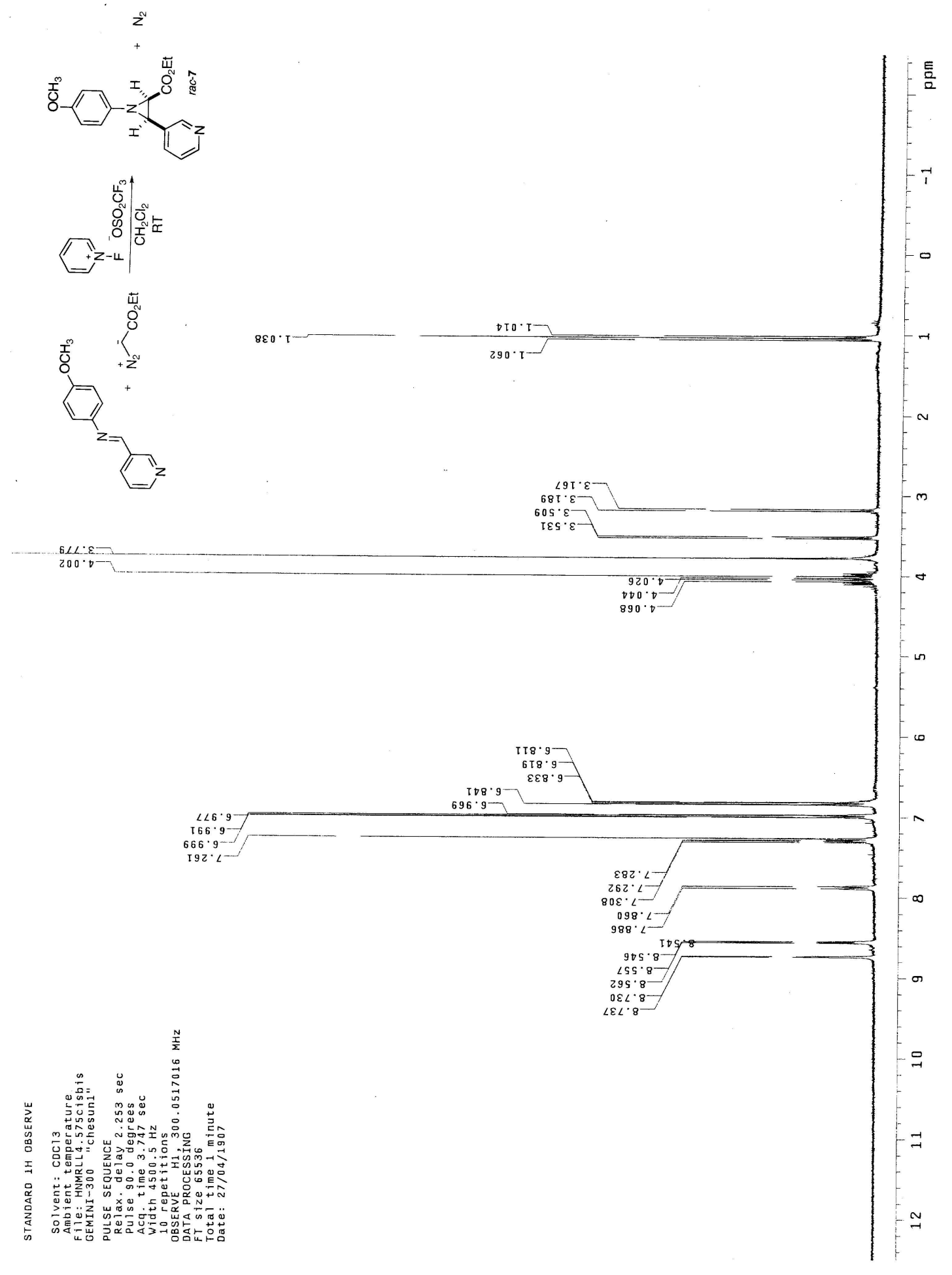


${ }^{13} \mathrm{C}-\mathrm{NMR}$ for $\mathrm{rac}-\mathbf{1 3}$

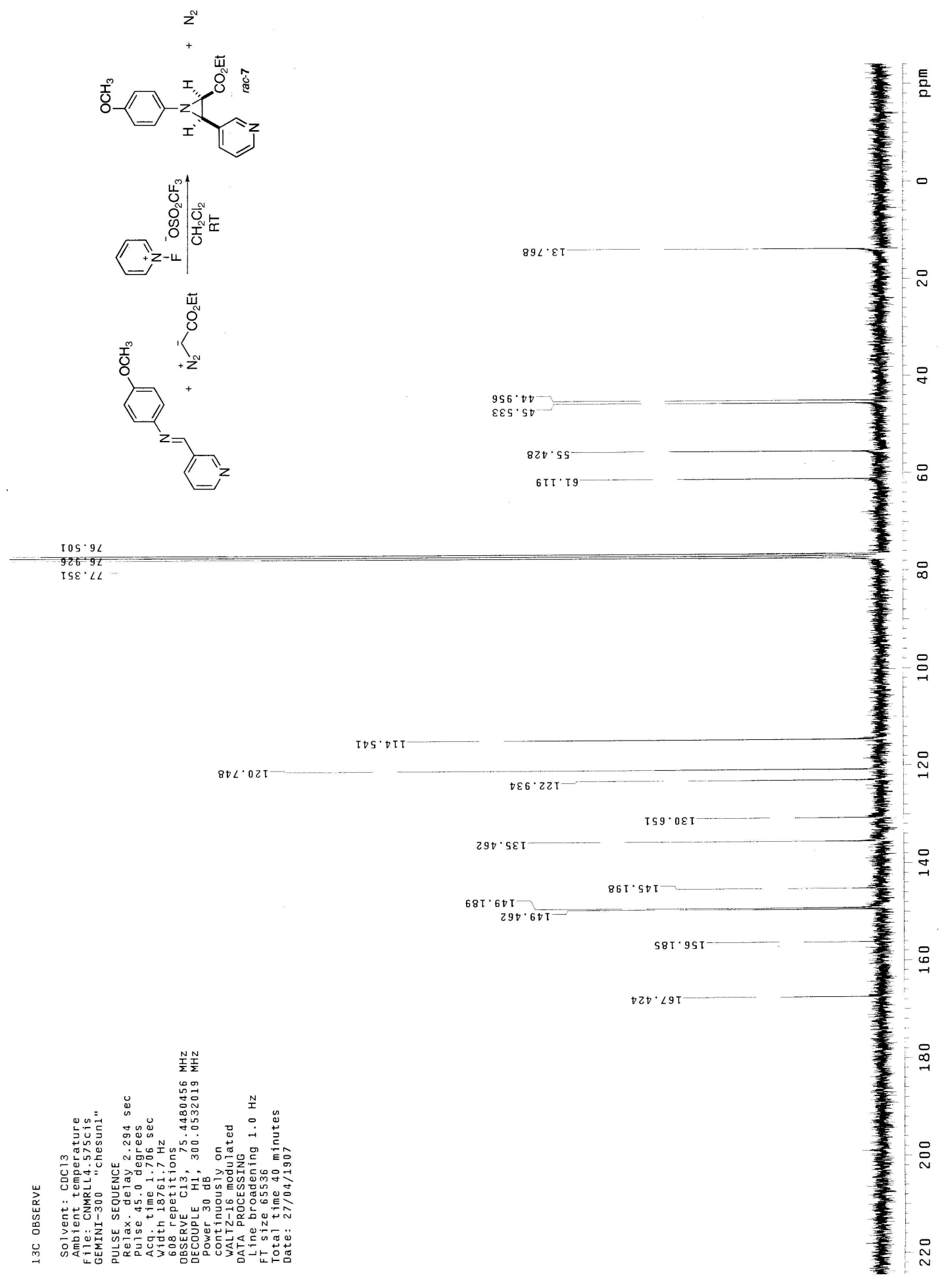




\section{${ }^{1} \mathrm{H}-\mathrm{NMR}$ for $\mathrm{rac}-14$}

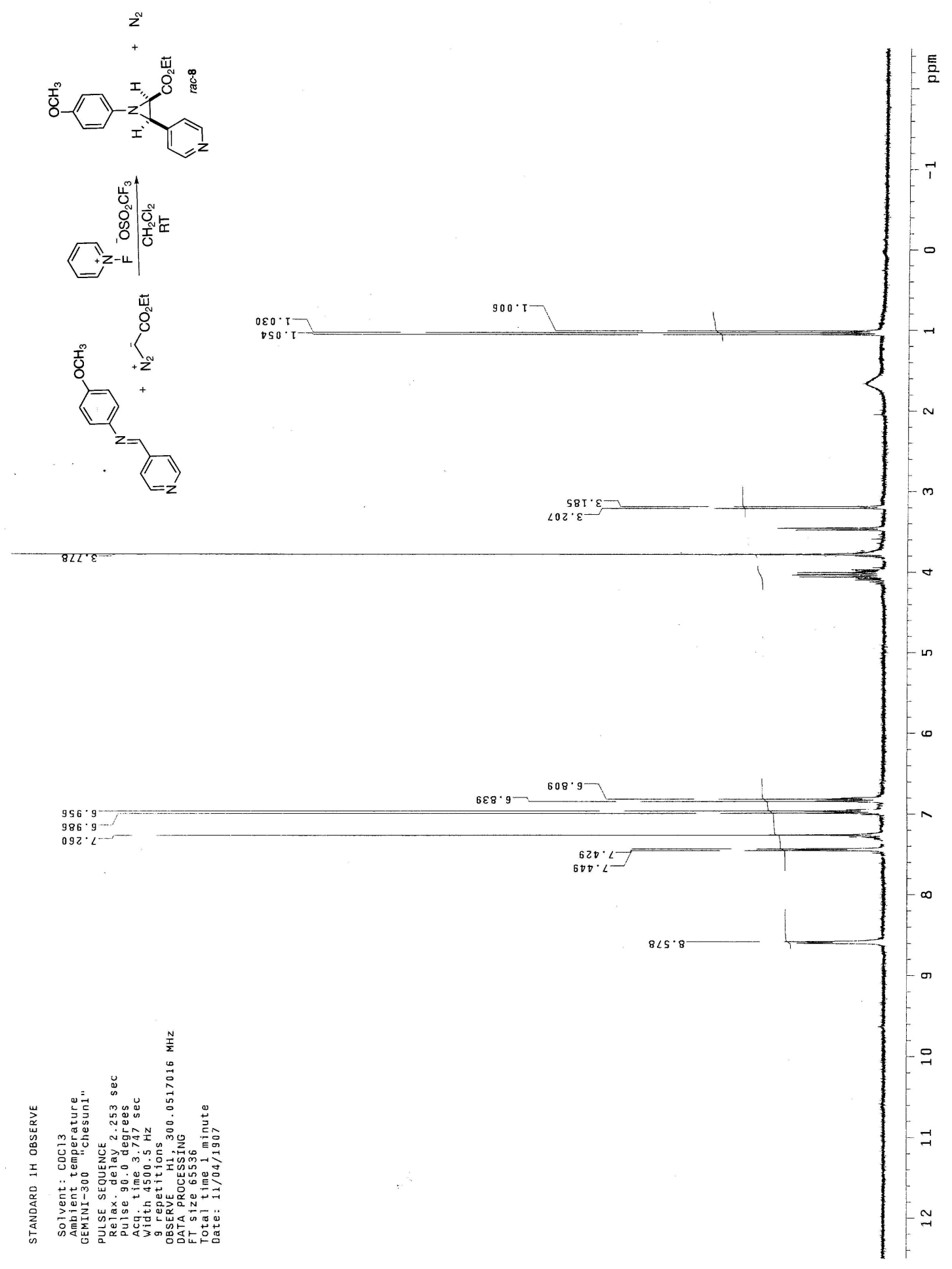


${ }^{13} \mathrm{C}-\mathrm{NMR}$ for $\mathrm{rac}-\mathbf{1 4}$

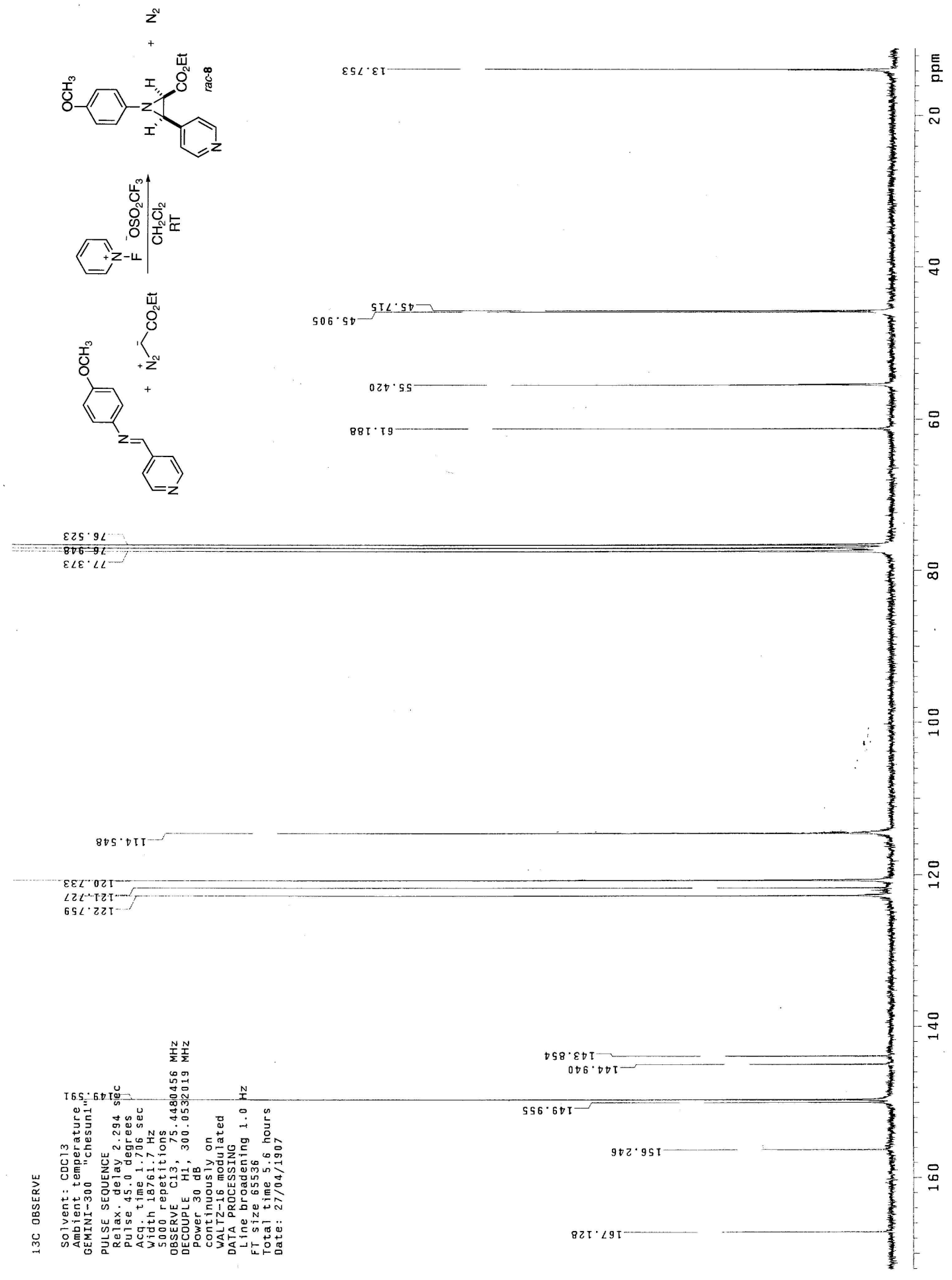




\section{${ }^{1} \mathrm{H}-\mathrm{NMR}$ for $\mathrm{rac}-\mathbf{1 5}$}

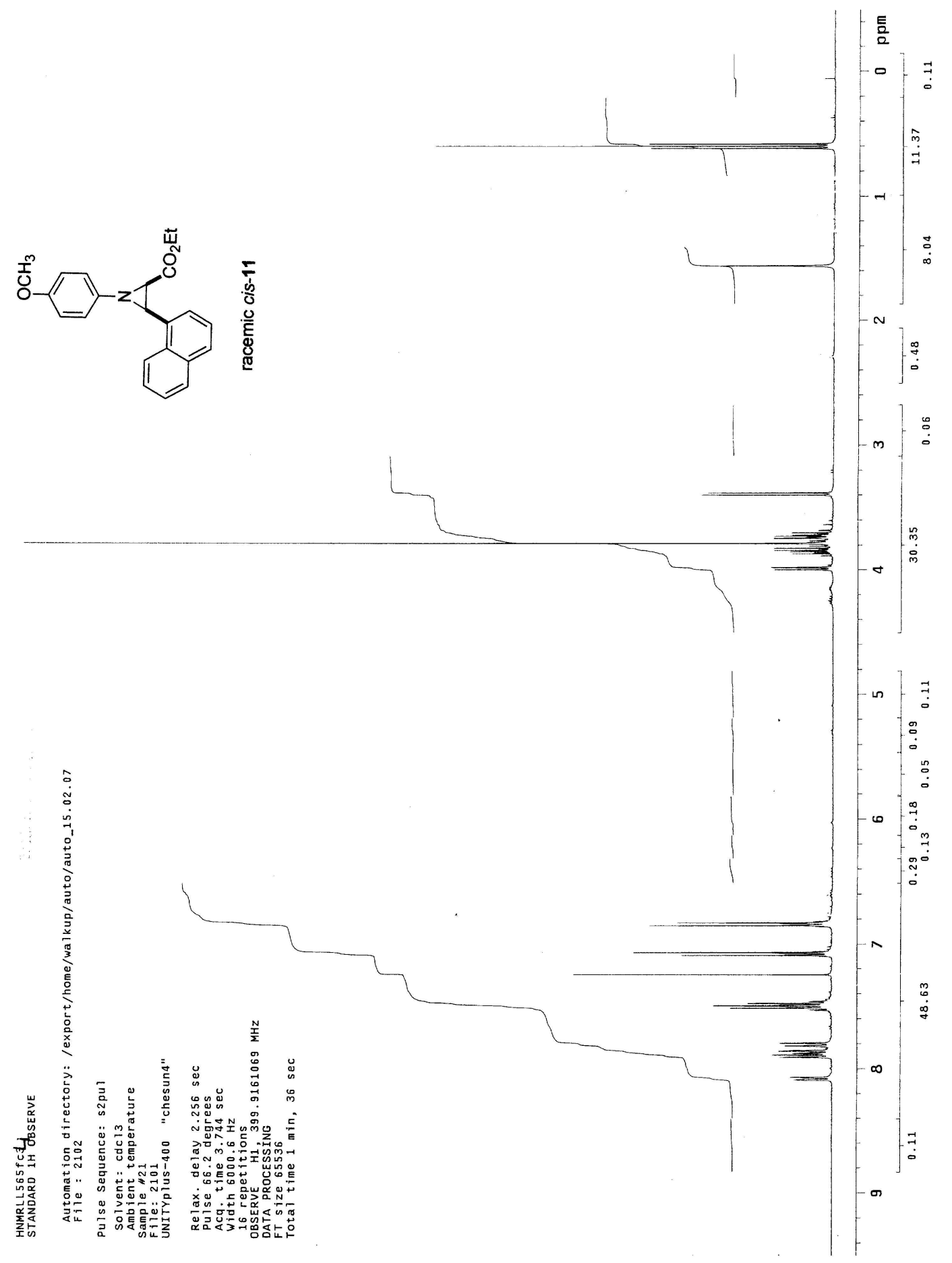




\section{${ }^{13} \mathrm{C}-\mathrm{NMR}$ for $r a c-15$}

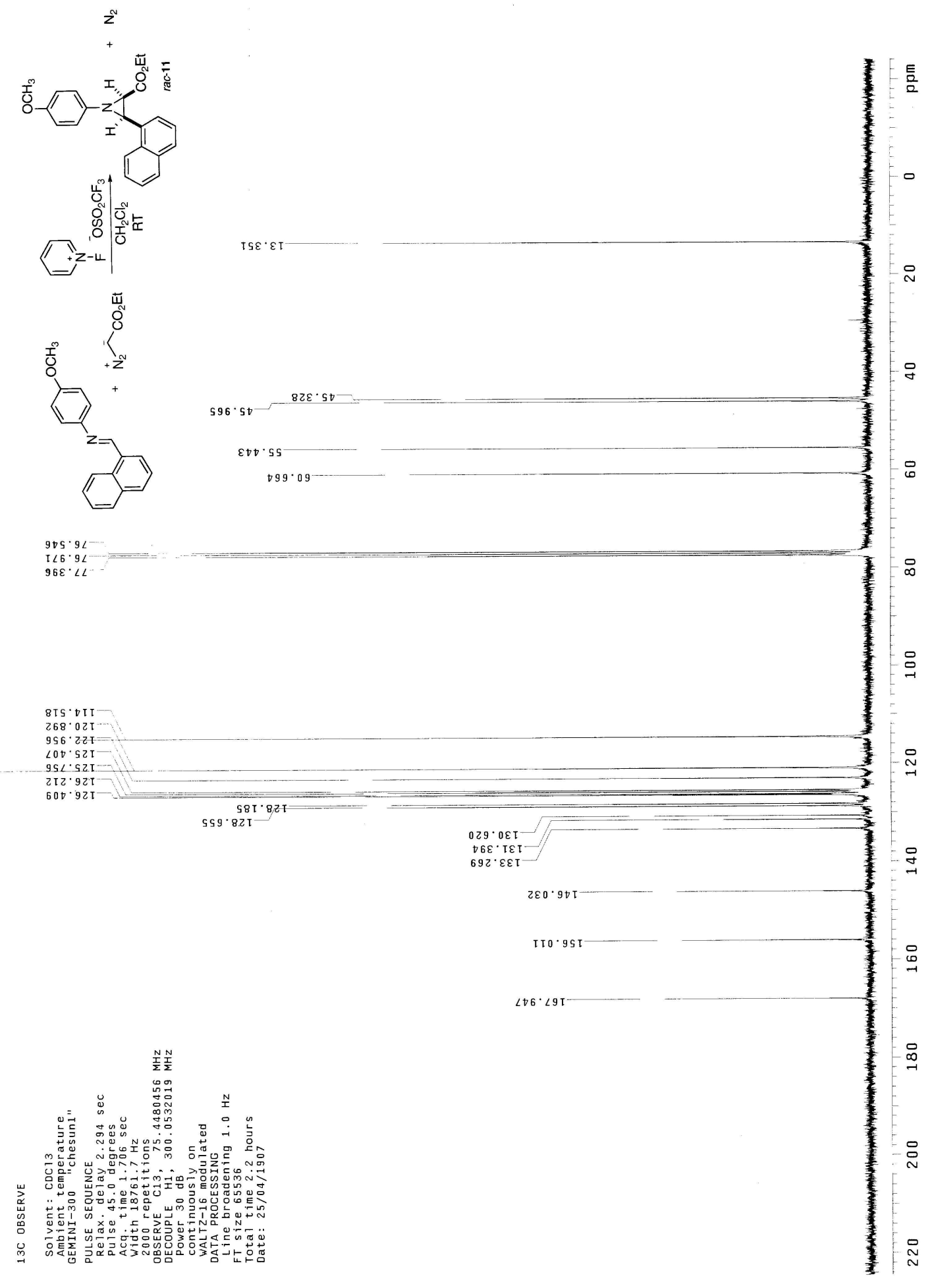


${ }^{1} \mathrm{H}-\mathrm{NMR}$ for $\mathrm{rac}-16$

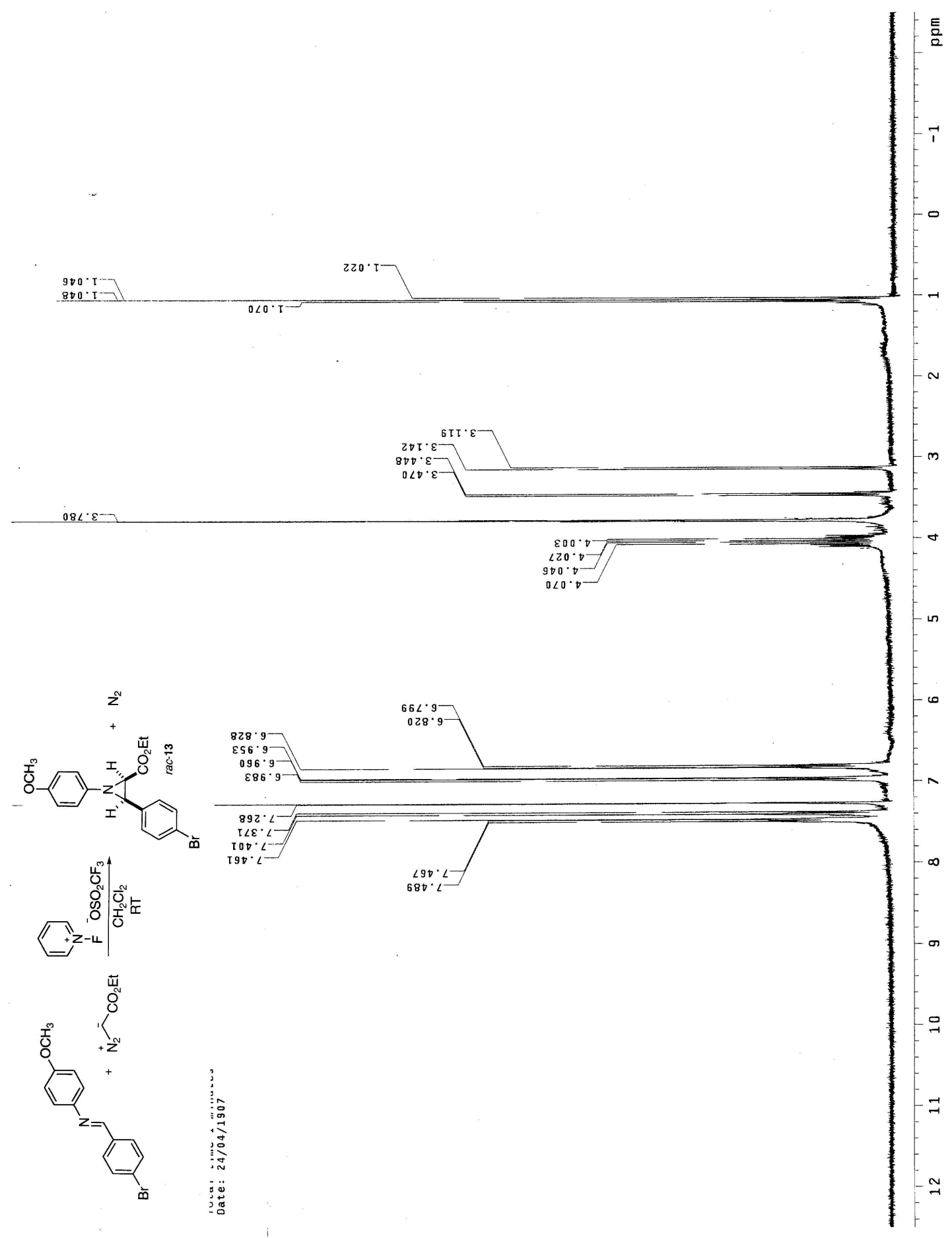


${ }^{13} \mathrm{C}-\mathrm{NMR}$ for $\mathrm{rac}-16$

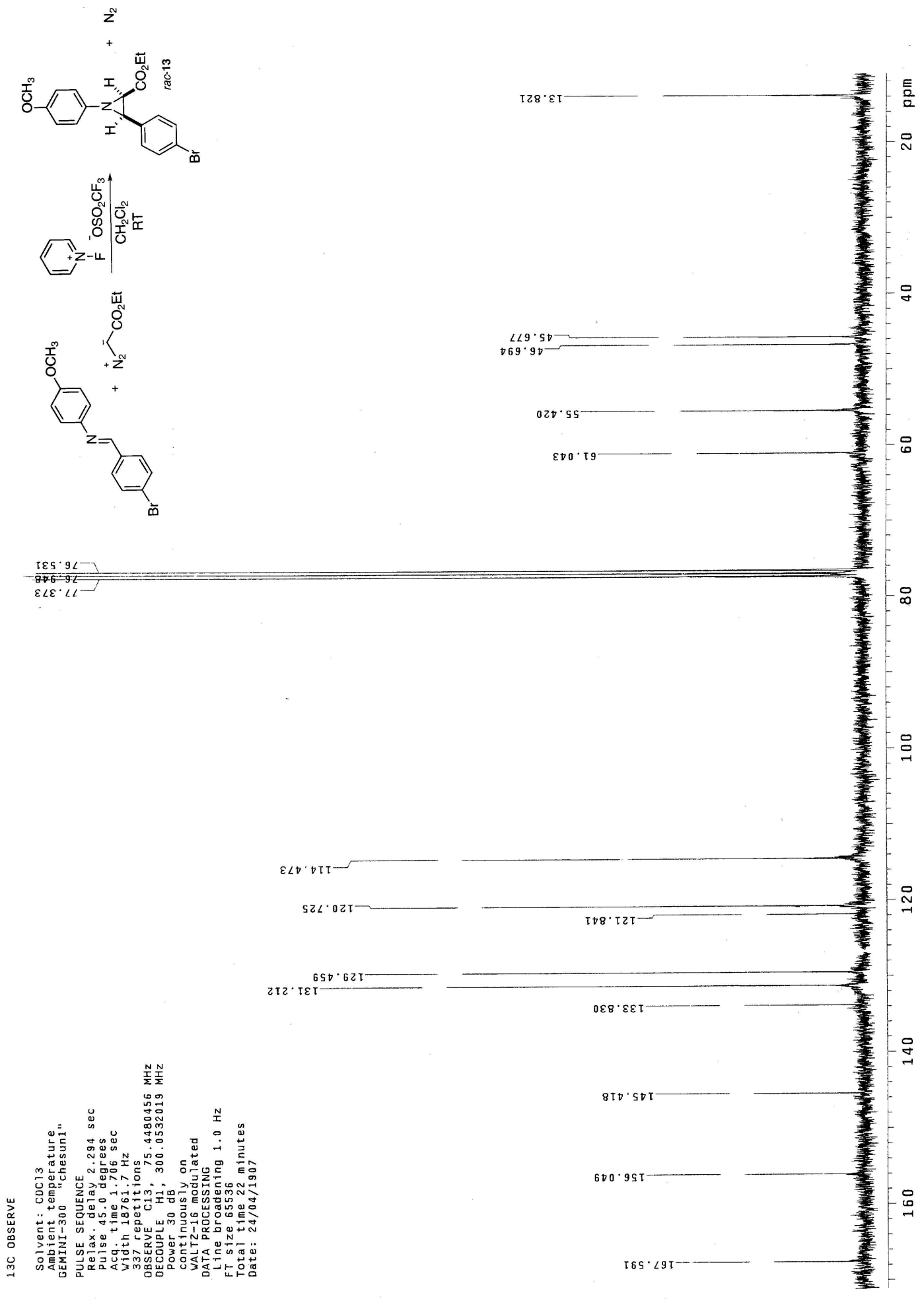




\section{${ }^{1} \mathrm{H}-\mathrm{NMR}$ for $\mathrm{rac}-\mathbf{1 7}$}

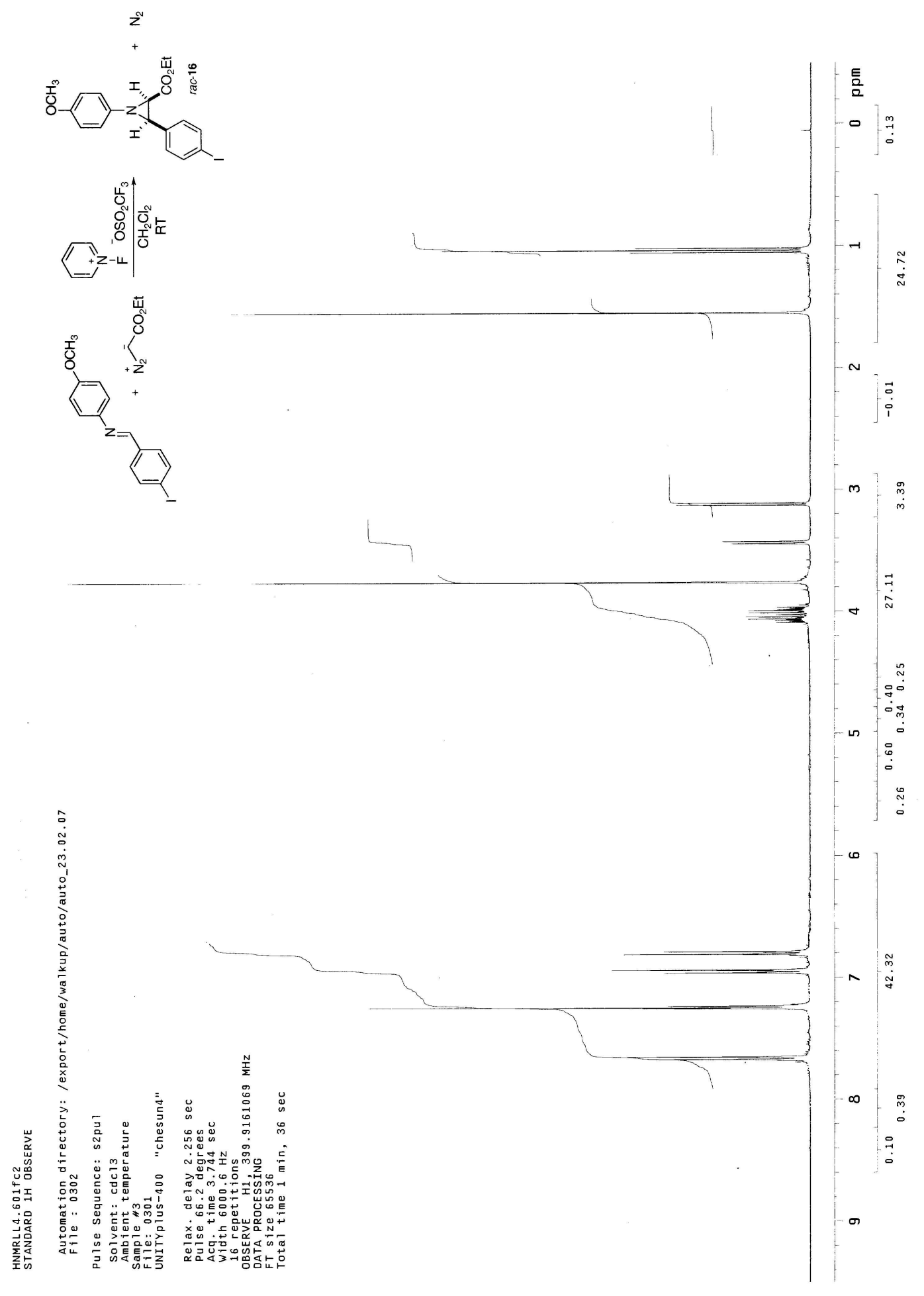


${ }^{13} \mathrm{C}-\mathrm{NMR}$ for $\mathrm{rac}-\mathbf{1 7}$

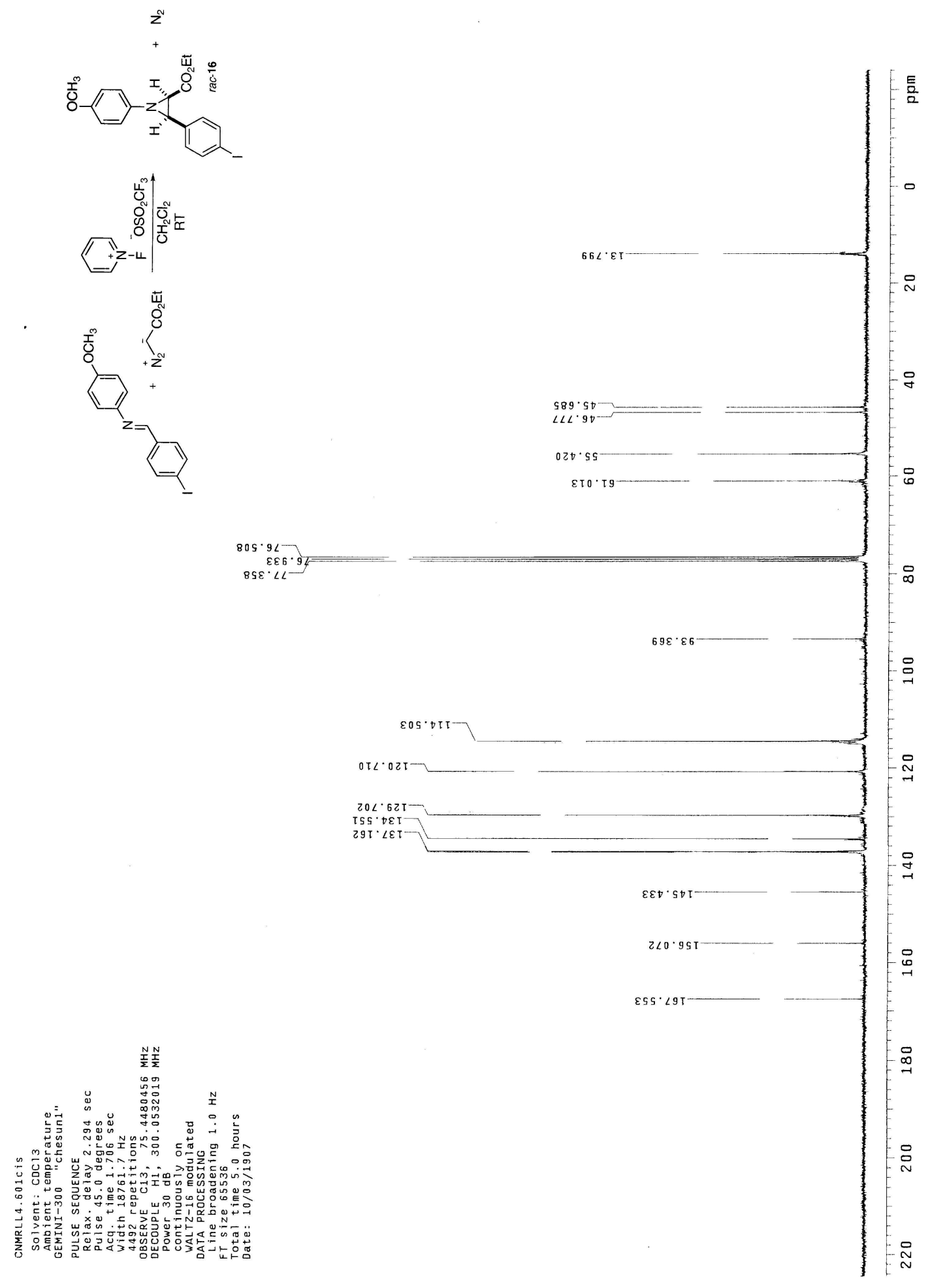




\section{${ }^{1} \mathrm{H}-\mathrm{NMR}$ for $r a c-18$}

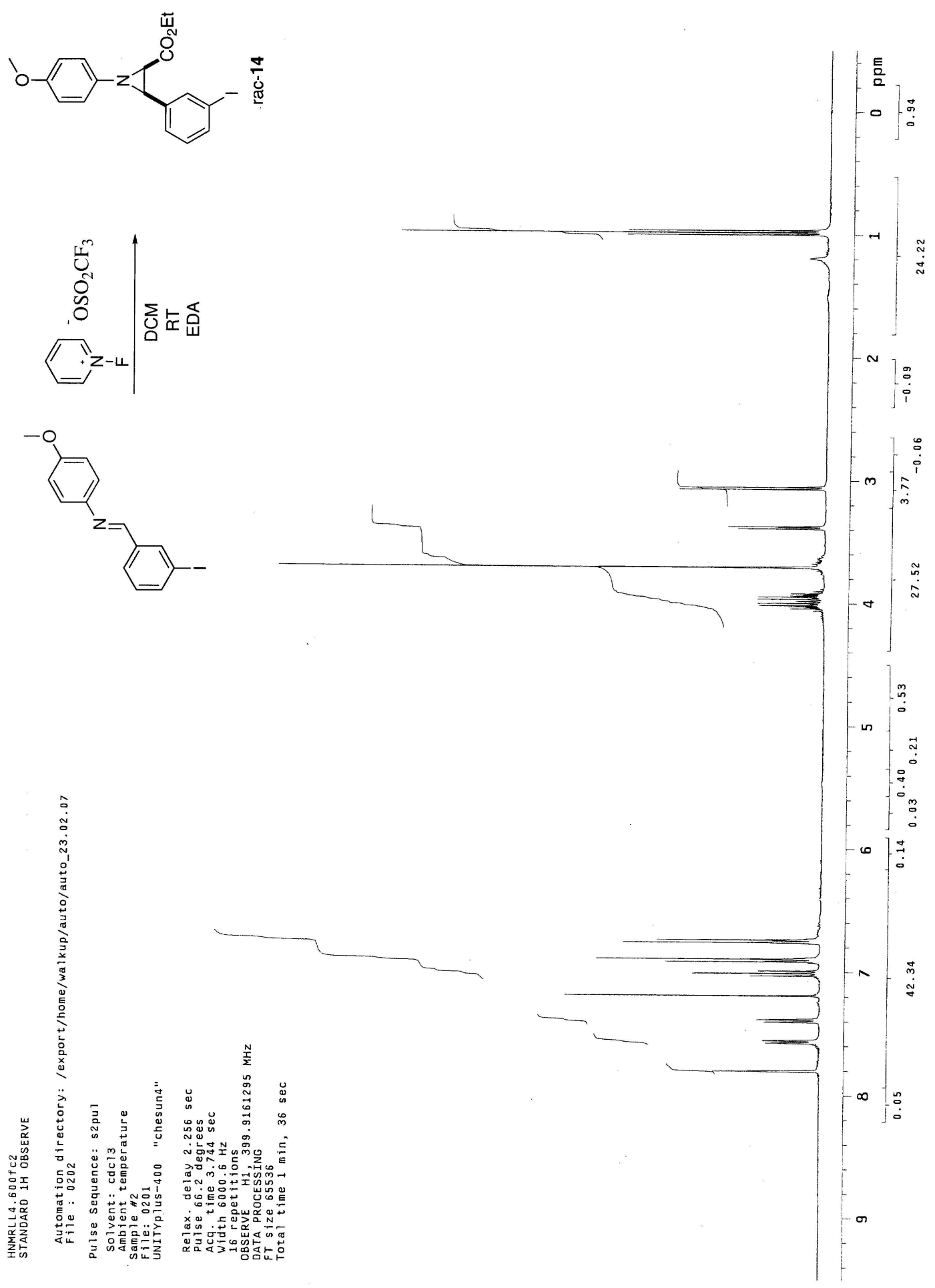




\section{${ }^{1} \mathrm{H}-\mathrm{NMR}$ for $r a c-19$}

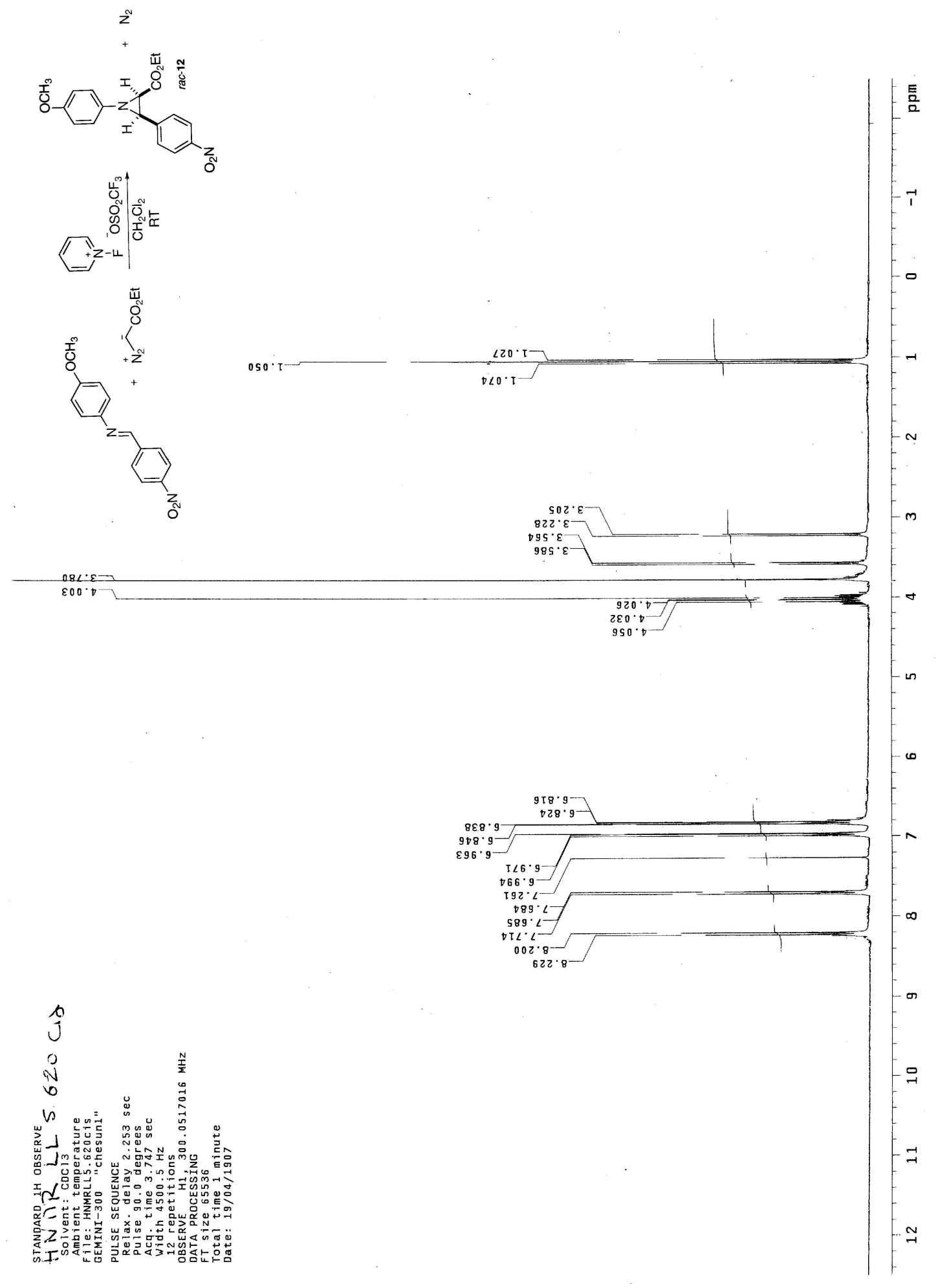


${ }^{13} \mathrm{C}-\mathrm{NMR}$ for $r a c-19$

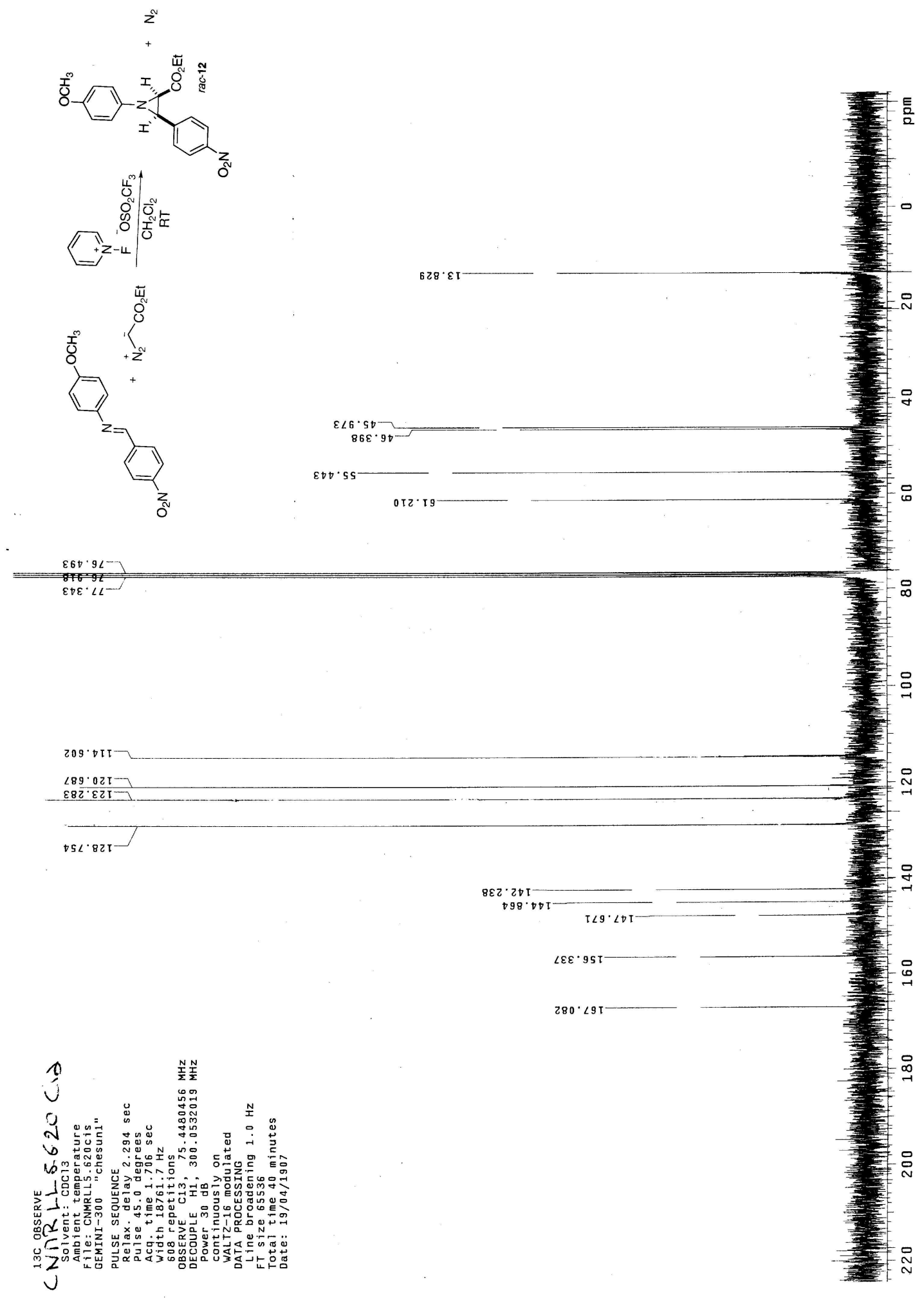


${ }^{1} \mathrm{H}-\mathrm{NMR}$ for $\mathrm{rac}-20$

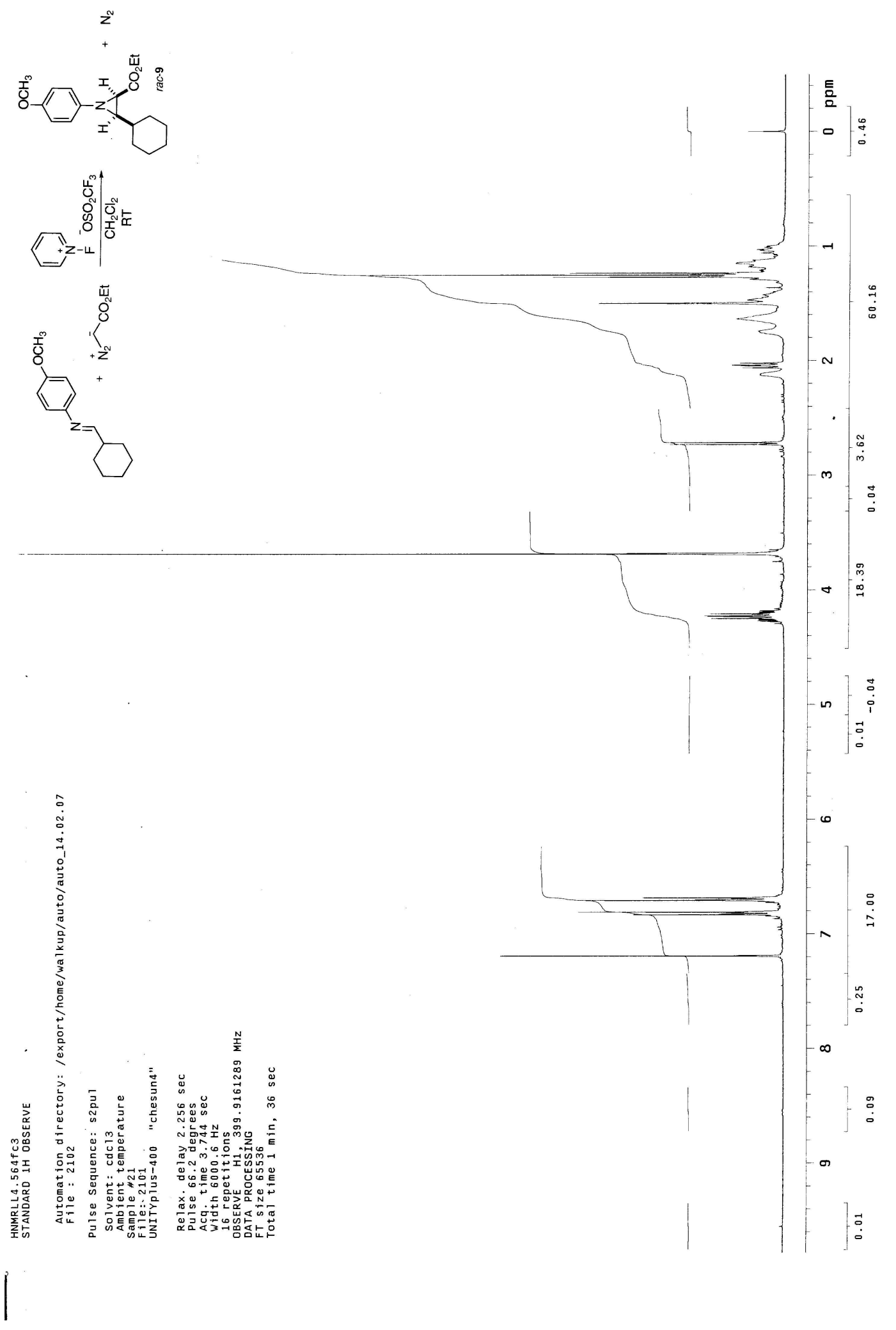


${ }^{13} \mathrm{C}-\mathrm{NMR}$ for $r a c-20$

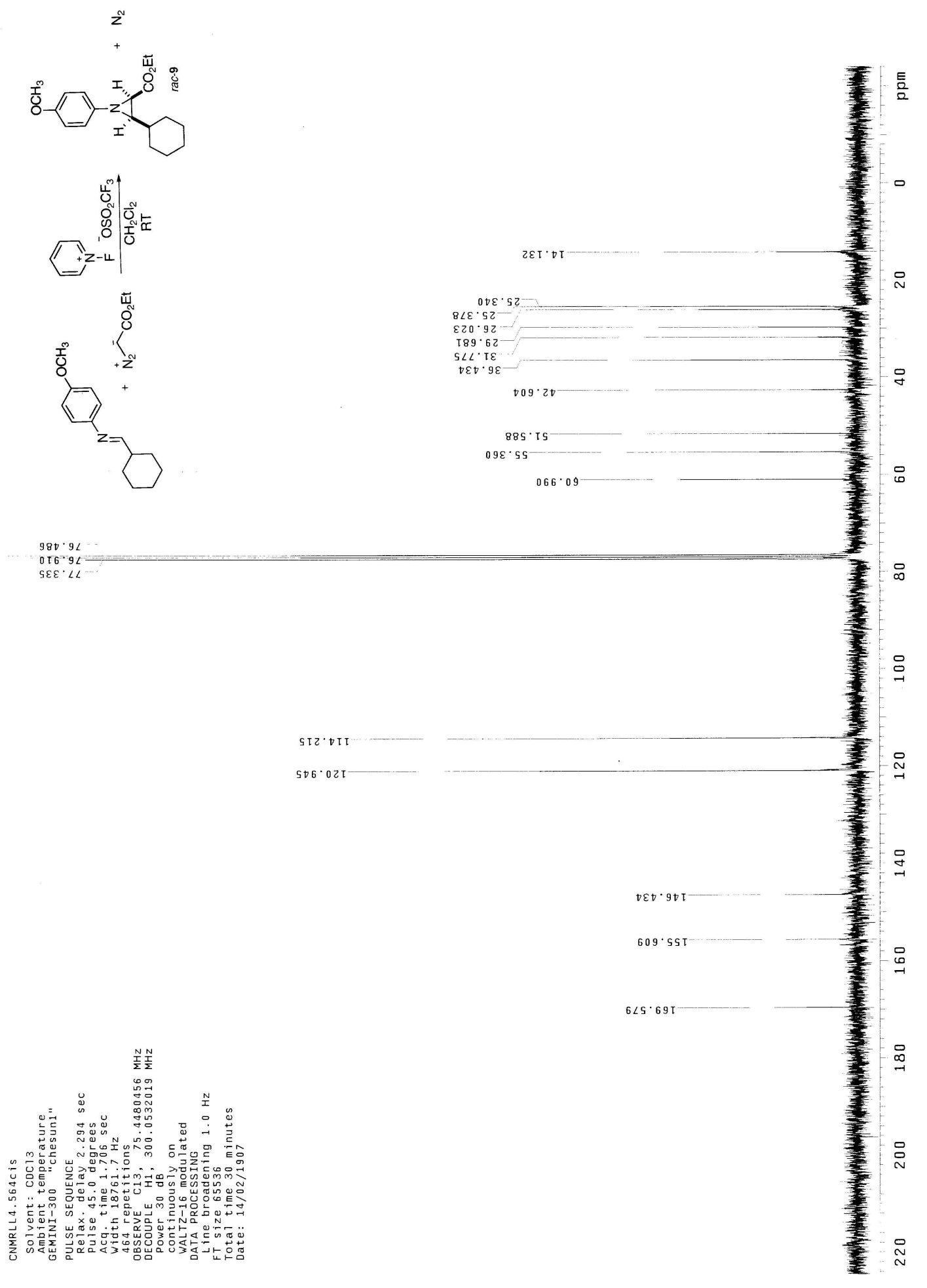




\section{${ }^{1} \mathrm{H}-\mathrm{NMR}$ for $r a c-21$}

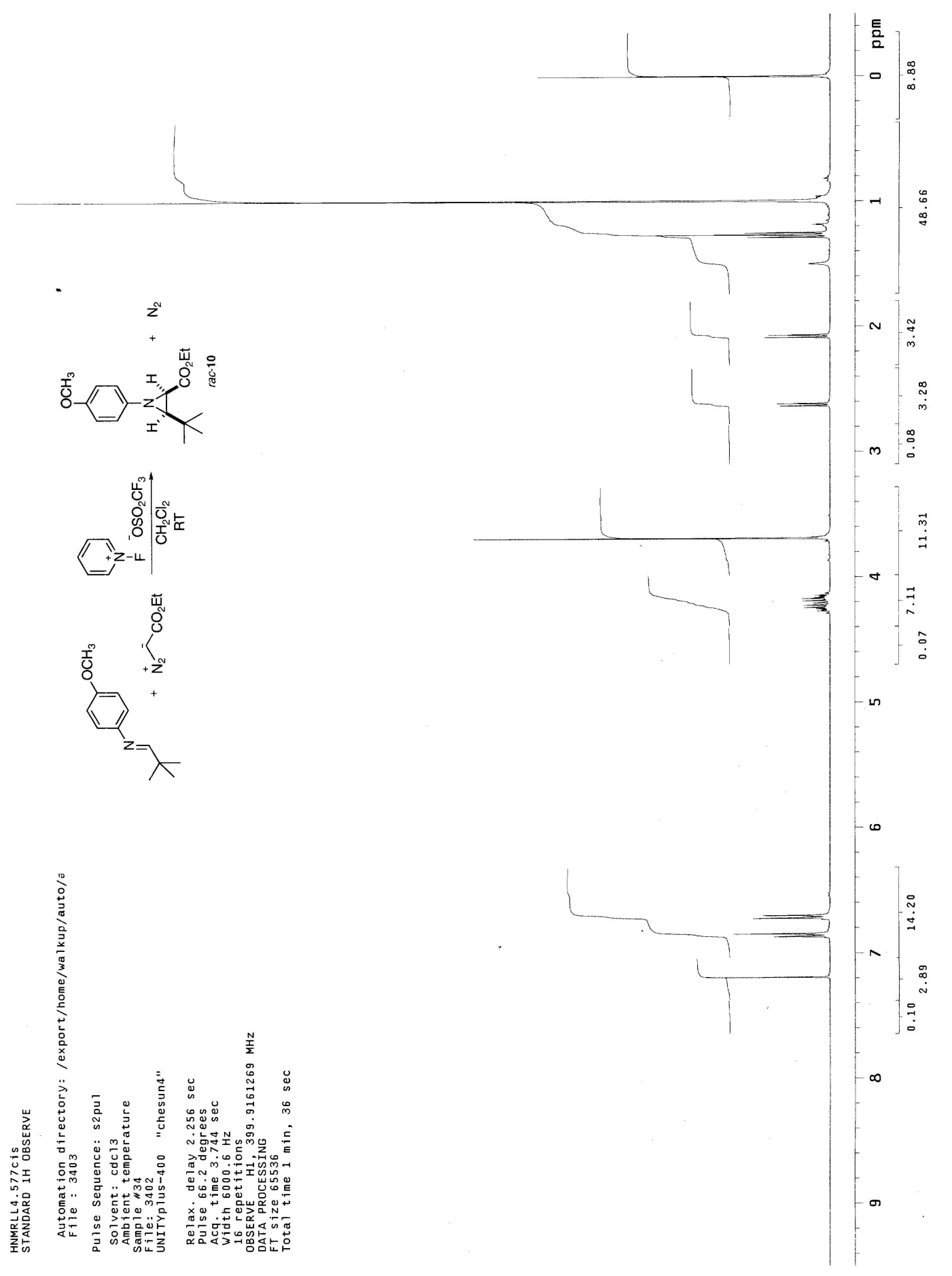


${ }^{13} \mathrm{C}-\mathrm{NMR}$ for $r a c-21$

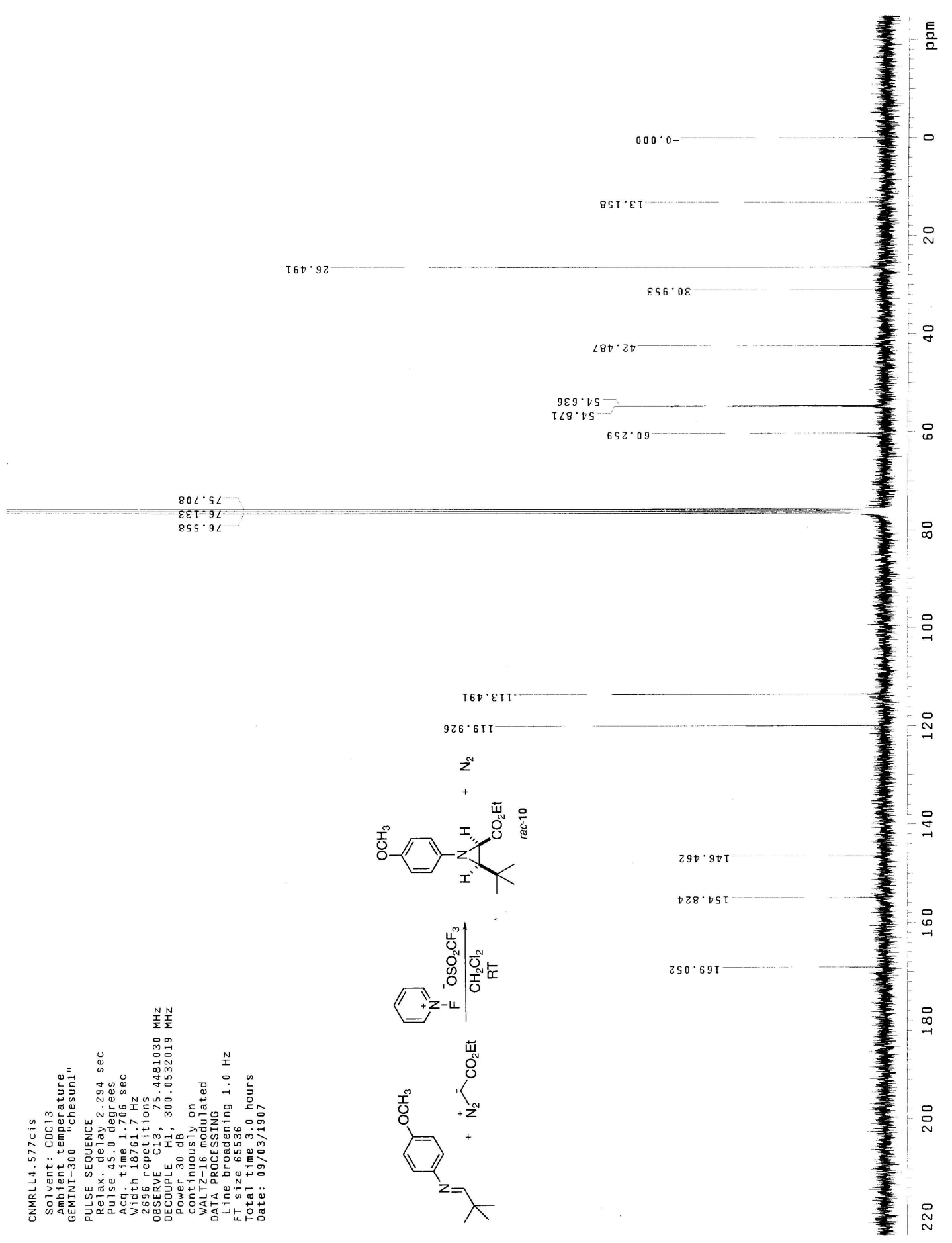


${ }^{1} \mathrm{H}-\mathrm{NMR}$ for rac-cis-23

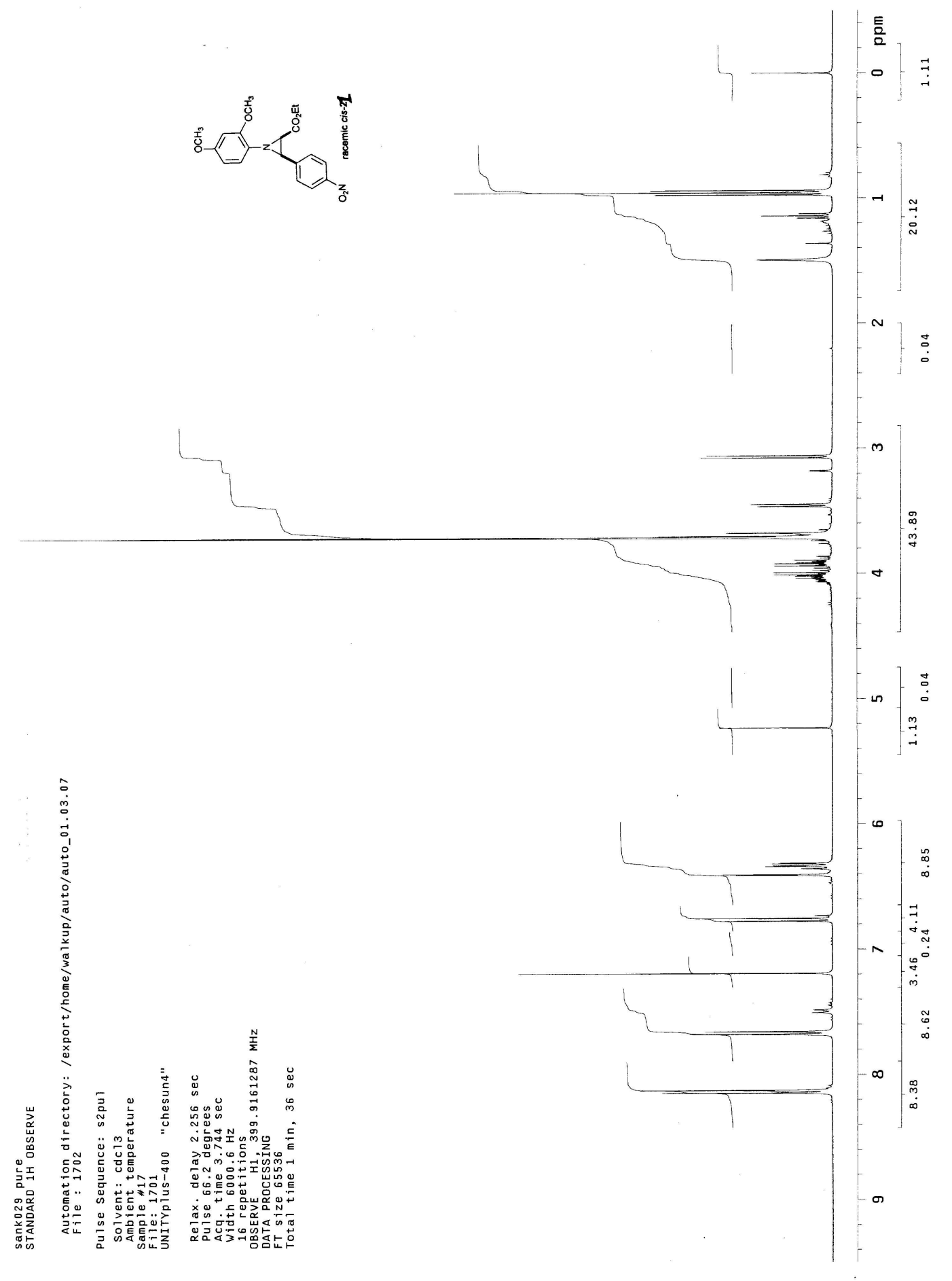


${ }^{13} \mathrm{C}-\mathrm{NMR}$ for $r a c-c i s-23$
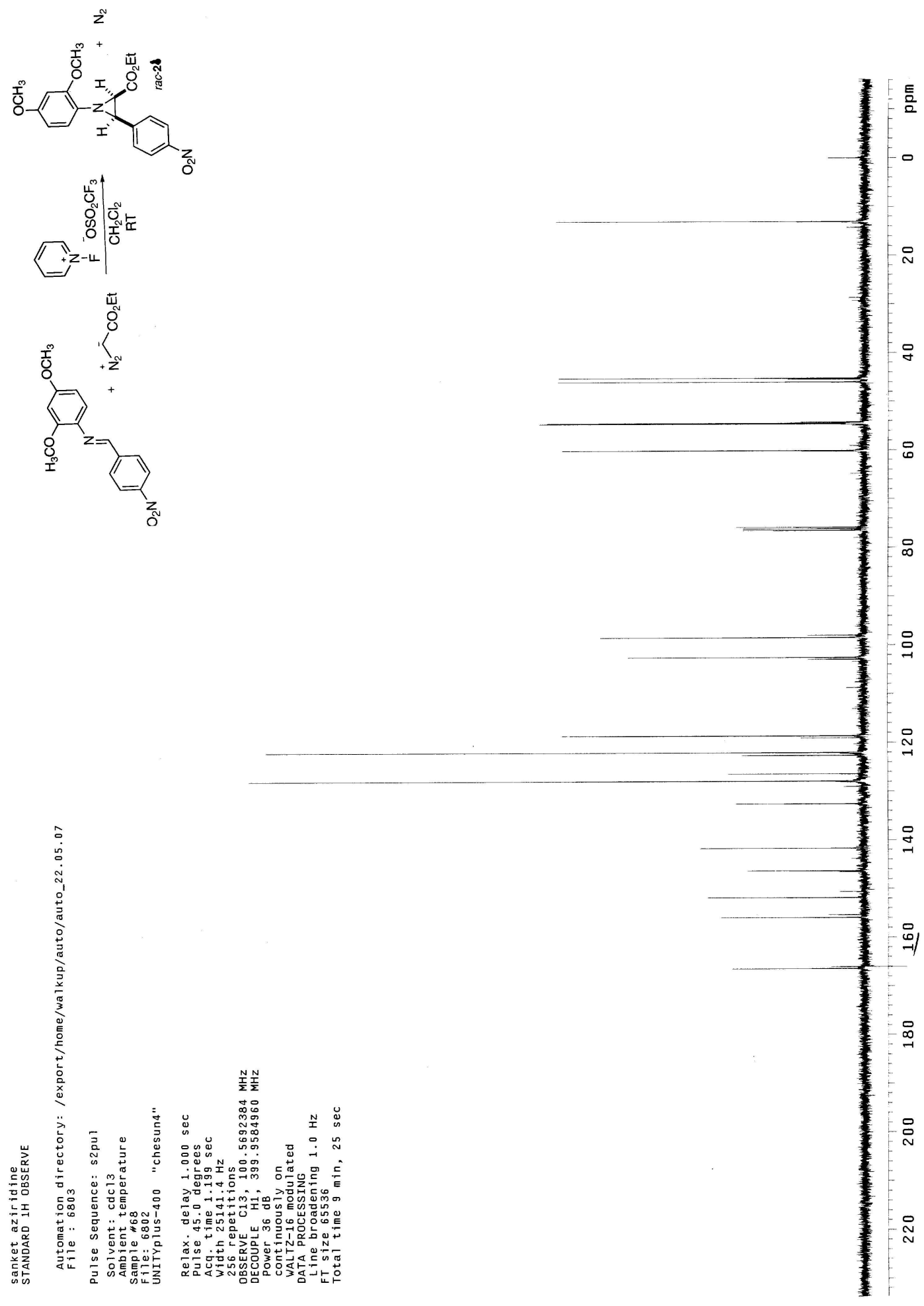


\section{${ }^{1} \mathrm{H}-\mathrm{NMR}$ for $\mathrm{rac}-27$}

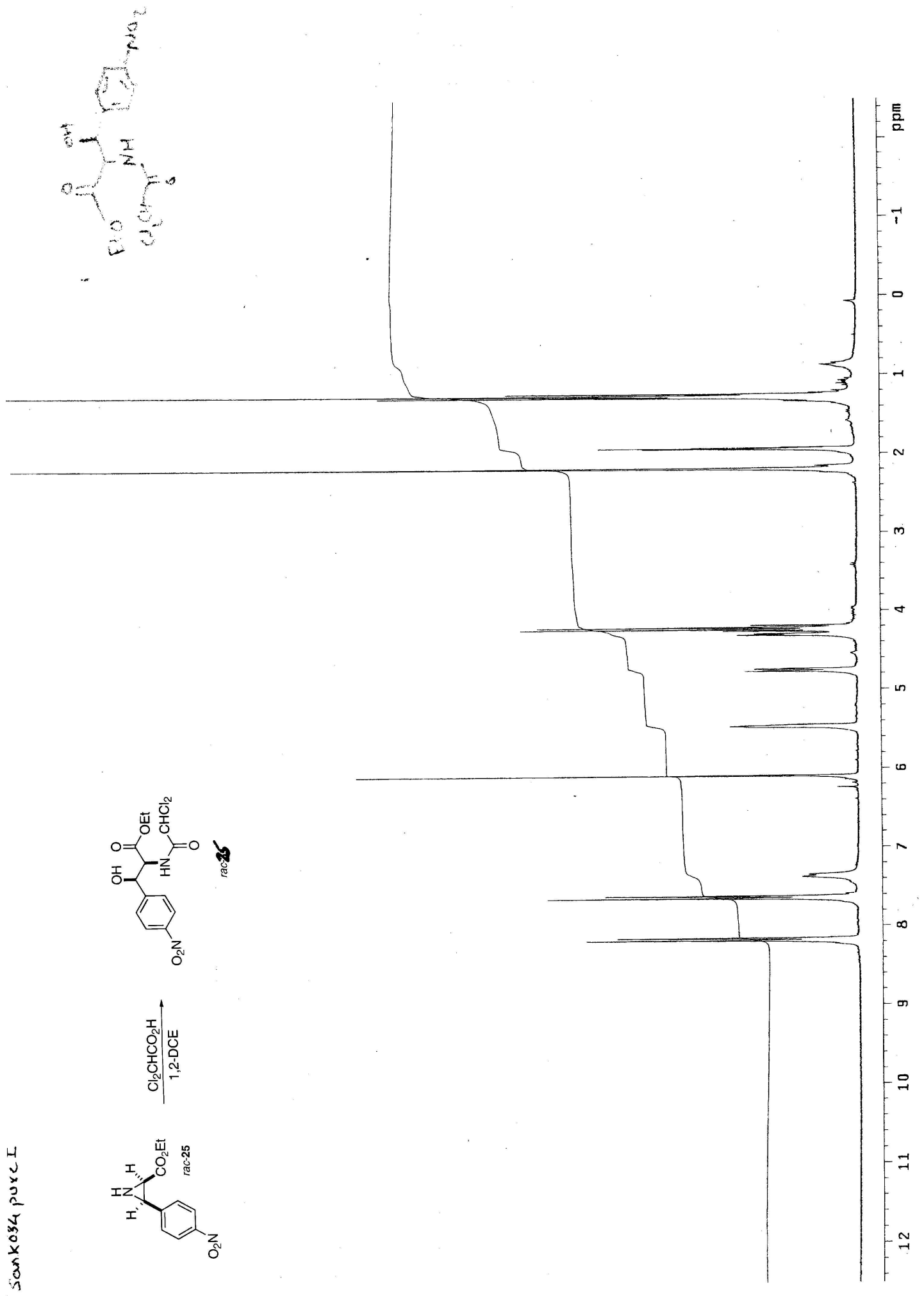


${ }^{13} \mathrm{C}-\mathrm{NMR}$ for $\mathrm{rac}-27$

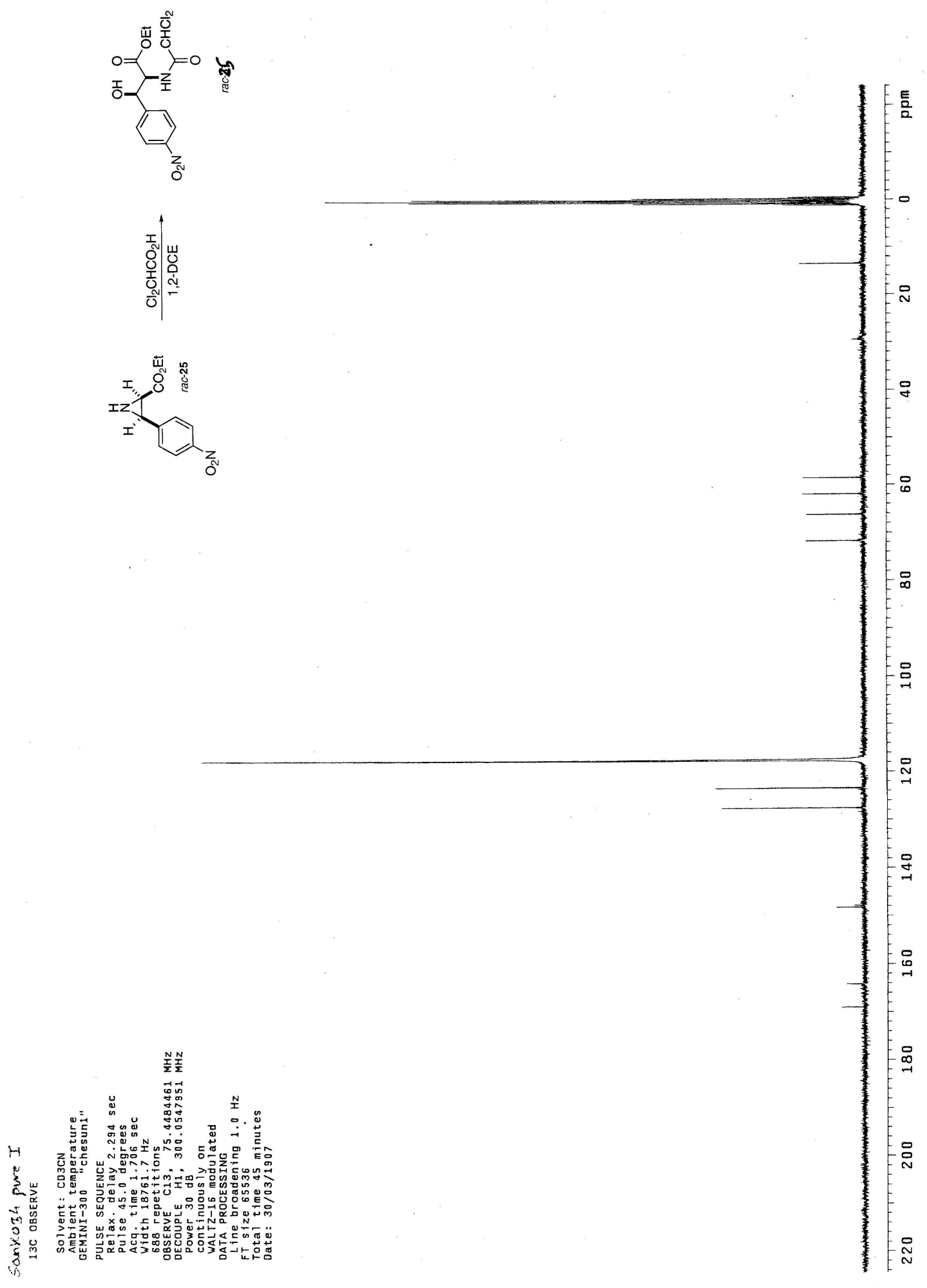




\section{${ }^{1} \mathrm{H}-\mathrm{NMR}$ for $r a c-28$}

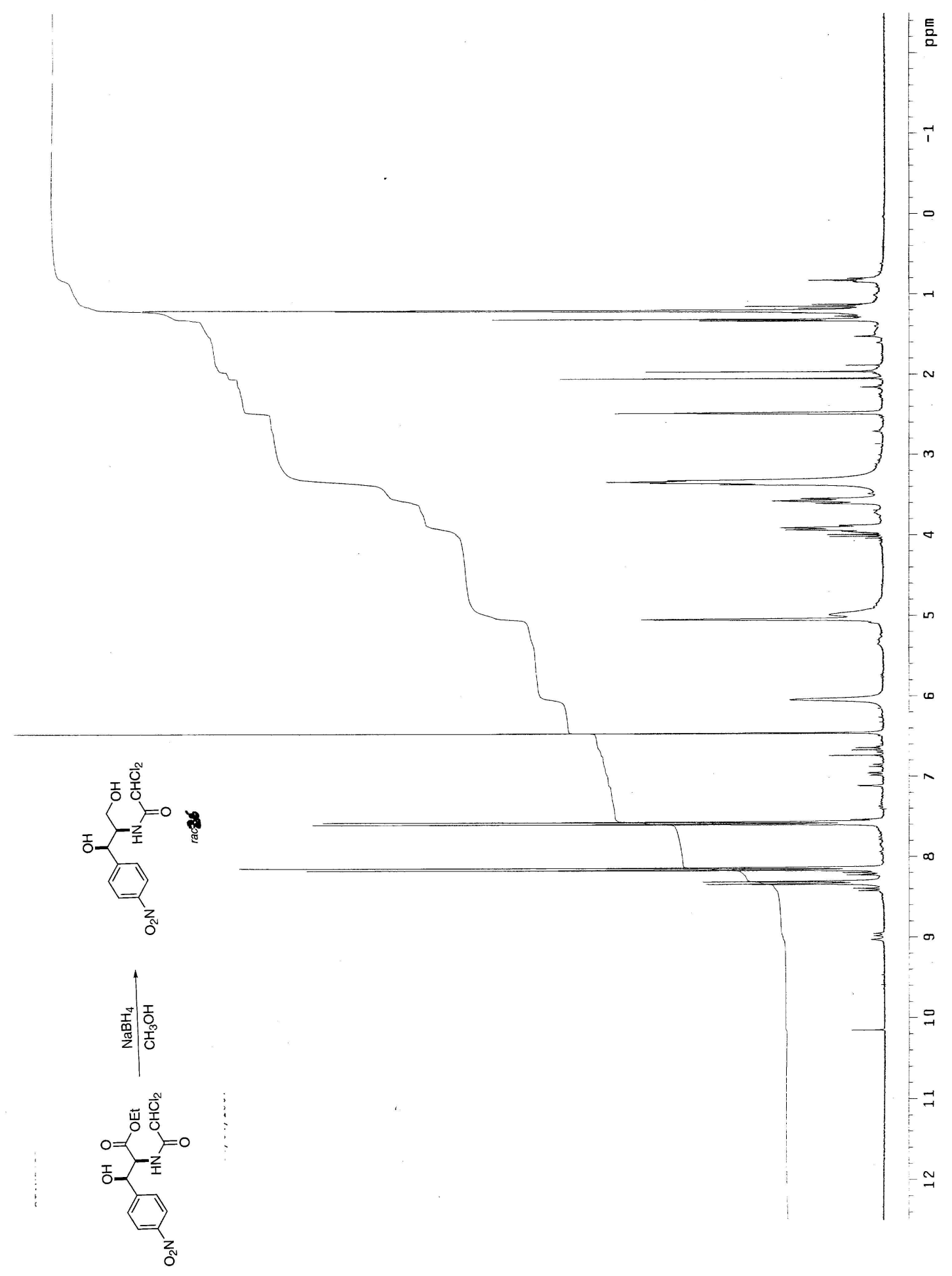


${ }^{13} \mathrm{C}-\mathrm{NMR}$ for $\mathrm{rac}-28$
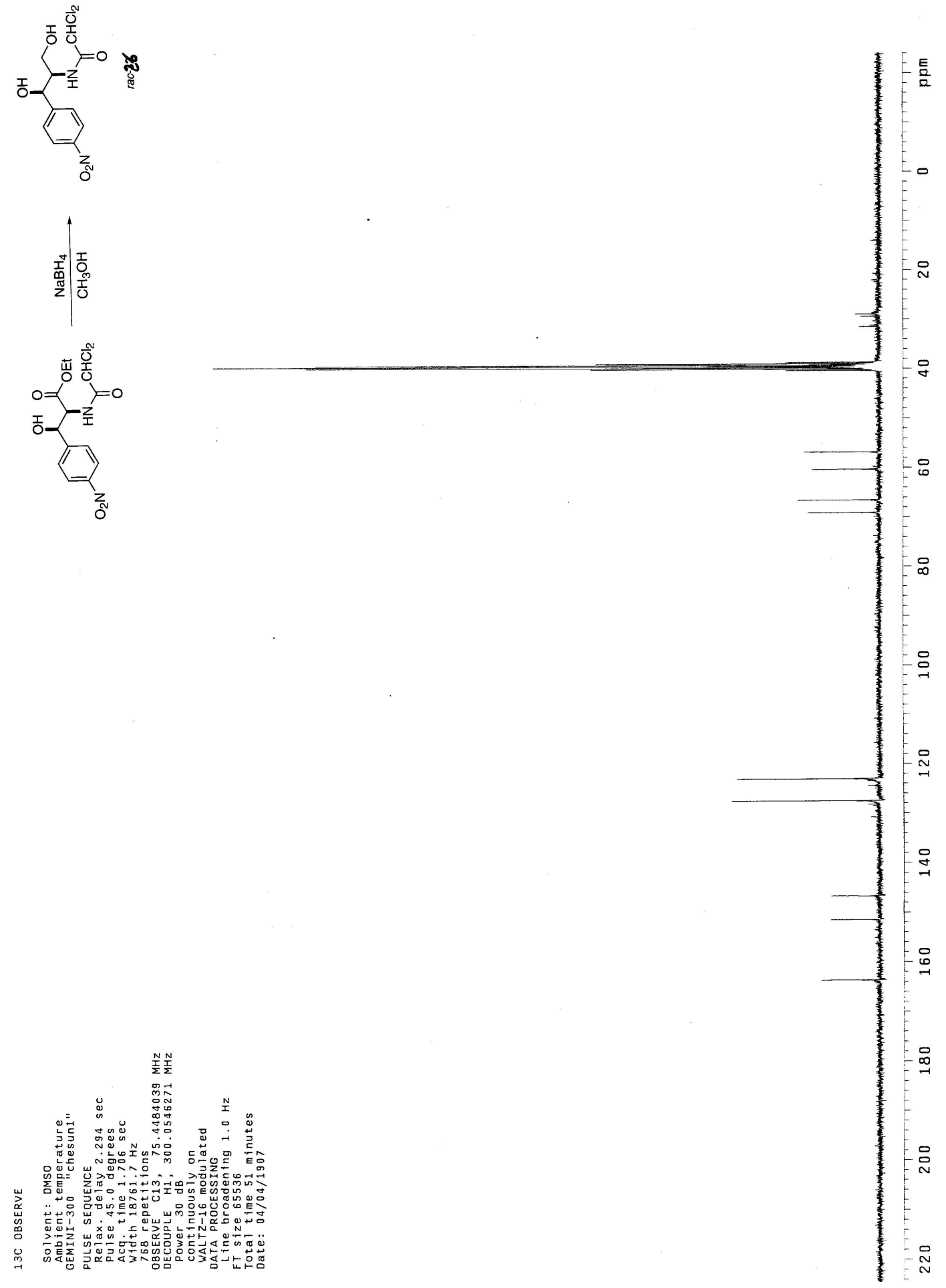
HRMS for N-H pyridinium triflate

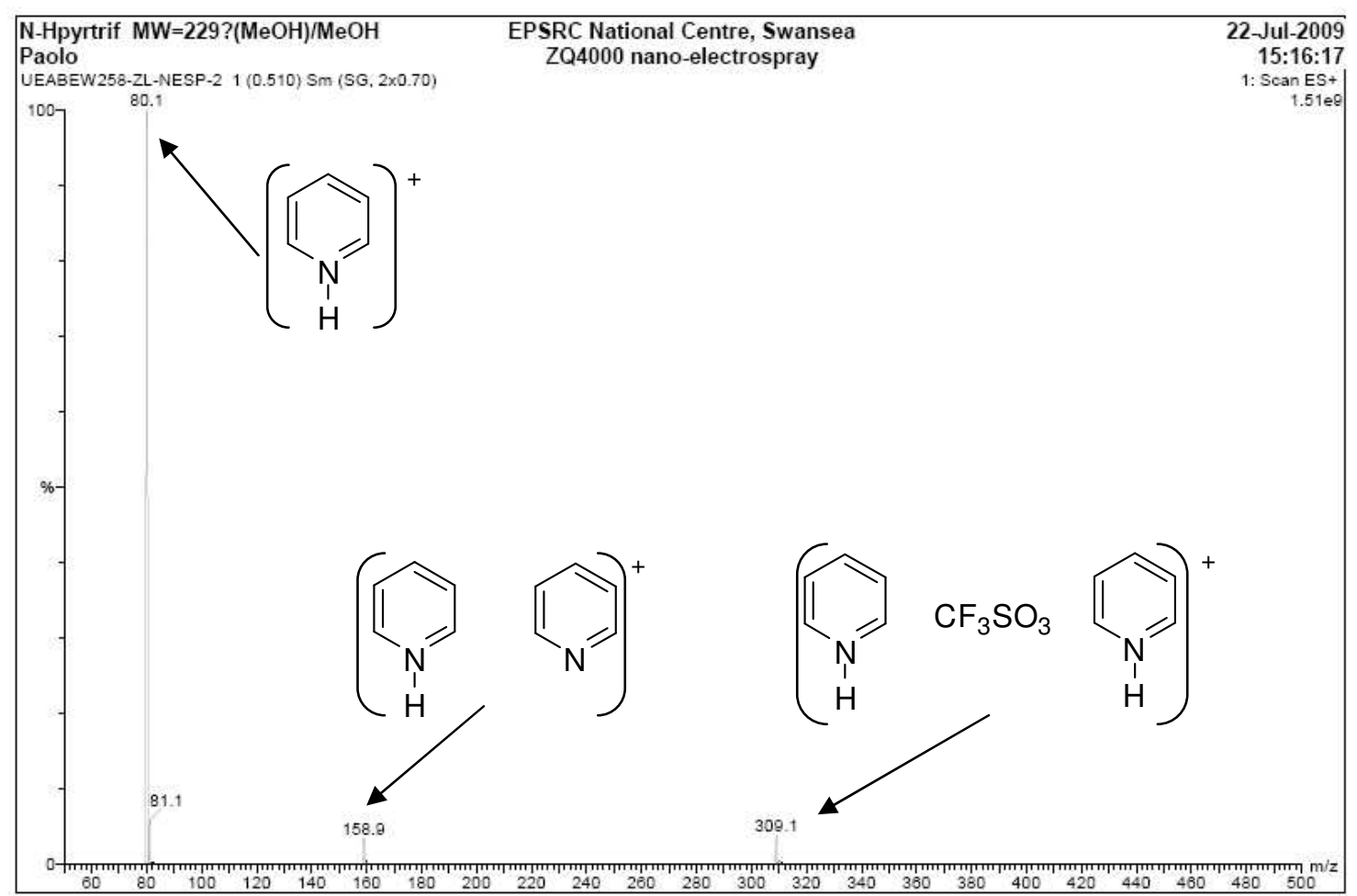

HRMS for N-fluoropyridinium triflate

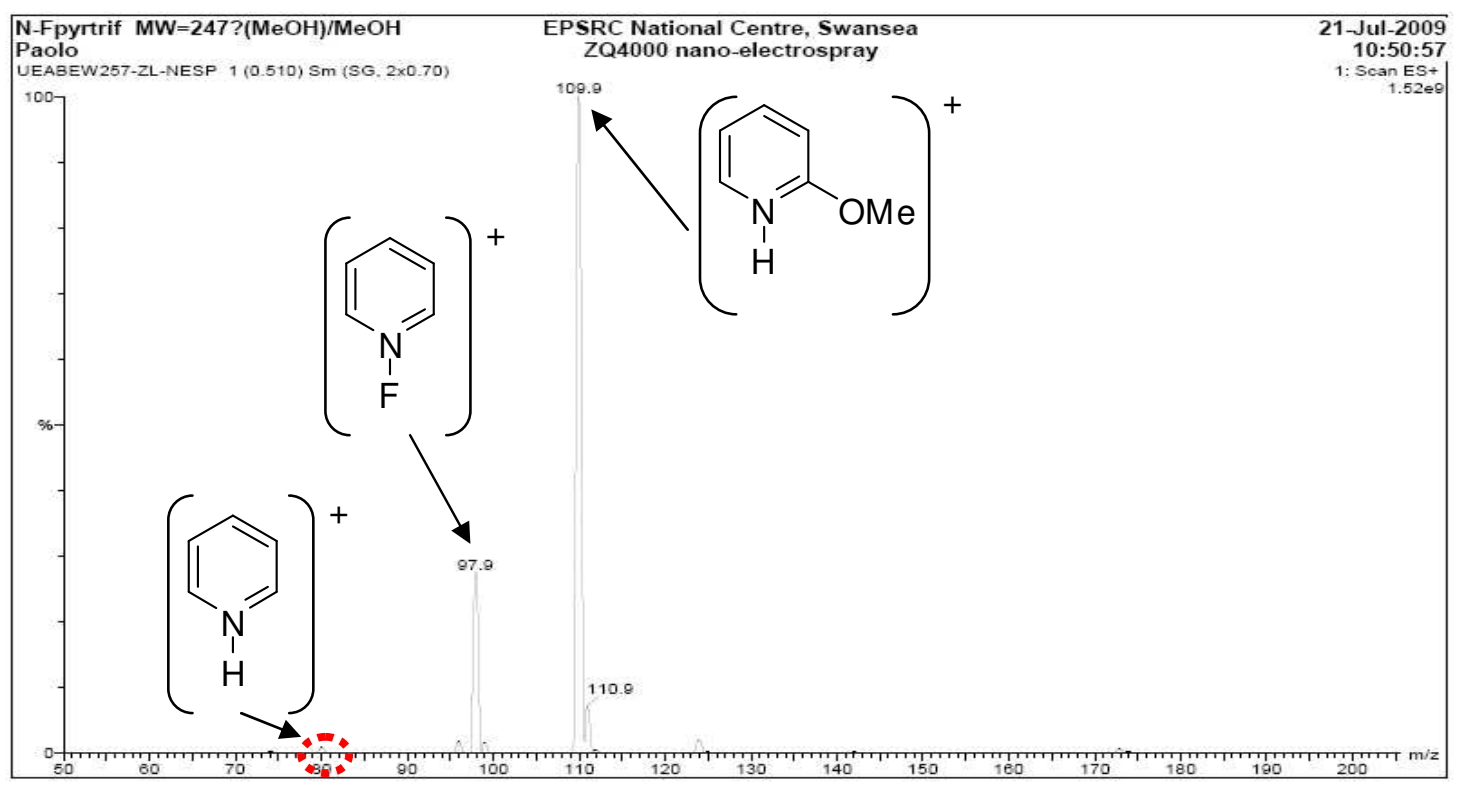

